\title{
Shedding light on the free radical nature of sulfonated melanins
}

J.V. Paulin a,b, A. Batagin-Neto ${ }^{\mathrm{a}, \mathrm{c}}$, E. Richards ${ }^{\mathrm{d}}$, P. Meredith ${ }^{\mathrm{b}, \mathrm{g}}$, C.F.O. Graeff ${ }^{\mathrm{a}, \mathrm{e}}$, A.B. Mostert ${ }^{\mathrm{f}}$

a São Paulo State University (UNESP), School of Sciences, Postgraduate Program in Science and Technology of Materials (POSMAT), Bauru, Brazil.

b Swansea University, Department of Physics, Singleton Park, Swansea, SA2 8PP, United Kingdom.

c São Paulo State University (UNESP), Campus of Itapeva, Itapeva, Brazil

${ }^{\mathrm{d}}$ Cardiff University, School of Chemistry, Cardiff, United Kingdom.

e São Paulo State University (UNESP), School of Sciences, Department of Physics, Bauru, Brazil.

f Swansea University, Department of Chemistry, Singleton Park, Swansea, SA2 8PP, United Kingdom.

g School of Mathematics and Physics, University of Queensland, St Lucia Campus, Brisbane Queensland 4072, Australia

Corresponding authors:

a.batagin@unesp.br (A. Batagin-Neto)

a.b.mostert@swansea.ac.uk (A.B.Mostert) 


\begin{abstract}
Melanin, an important class of natural pigment found in the human body, has stood out as a promising bioelectronic material due to its rather unique collection of electrical properties and biocompatibility. Among the available melanin derivatives, the sulfonated forms has proven to not only be able to produce homogeneous device quality thin-films with excellent adhesion even on hydrophobic surfaces, but also to act as an ion to electron transducing element. It has recently been shown that the transport physics (and dominat carrier generation) may be related to a semiquinone free radical species in these materials. Hence, a better understanding of the paramagnetic properties of sulfonated derivatives could shed light on their charge transport behavior, and thus enable improvement in regard use in bioelectronics. Motivated by this question, in this work, different sulfonated melanin derivatives were investigated by hydration-controlled continuous-wave X-band electron paramagnetic resonance spectroscopy and electronic structure calculations. Our results show that sulfonated melanin behaves similarly to non-functionalized melanin, but demonstrates a less pronounced response to humidity vis-à-vis standard melanin. We thus speculate on the structural and charge transport behavior in light of these differences with a view to further engineering structure-property relationships.
\end{abstract}

Keywords: Melanin; eumelanin; melanin derivatives; electron paramagnetic resonance; bioelectronics. 


\section{Introduction}

The transduction of an ionic signal to an electronic signal using an organic electronic device is a matter of central significance in the field of bioelectronics. One way to achieve transduction is by fabricating devices that are able to interface and amplify detected ionic signals by incorporating materials that can sustain both electronic and protonic/ionic signals. ${ }^{1-3}$

A promising material to act as such an interface is the biomacromolecule eumelanin. ${ }^{4,5}$ Eumelanin, or more commonly referred to simply as melanin, ${ }^{6}$ is the primary natural pigment of the melanic family of compounds. Melanin is a mix of heterogeneous oligomers stacked via $\pi-\pi$ interactions, with the oligomer sheets comprising of up to tens of moieties of 5,6-dihydroxyindole (DHI) and 5,6dihydroxyindole-2-carboxylic acid (DHICA) and their various redox forms (Figure 1a). ${ }^{7}$ Melanin has attracted attention due to its physicochemical properties such as metal ion chelation, ${ }^{8}$ radical scavenging, ${ }^{9}$ redox activity, ${ }^{10,11}$ inherent biocompatibility, ${ }^{12}$ a featureless exponential absorption in the UV and visible ranges, ${ }^{13}$ conductivities ranging from $10^{-8}$ to $10^{-4} \mathrm{Scm}^{-1}$ depending on its hydration state with an assumed mixed ionic/electronic charge transport ${ }^{14-17}$ and the presence of a stable free radical electron paramagnetic resonance (EPR) signal. ${ }^{18-20}$

In attempts to improve the understanding of melanin's properties, many researchers have resorted to making functionalized DHI and DHICA monomers and polymers. ${ }^{21-29}$ One of these functionalized polymers, pioneered by Graeff et al., is obtained through the oxidation of the precursor DL-(3,4-dihydroxyphenyl)-alanine (DL-DOPA) in dimethylsulfoxide (DMSO). ${ }^{21}$ Using this procedure, sulfonated groups are incorporated in the underlying DHI and DHICA monomers (Figure 1b), yielding a sulfonated melanin. ${ }^{30}$ The result is a derivative with higher thermal stability, ${ }^{21}$ biocompatibility, ${ }^{31}$ solubility in DMSO, N-methyl-2-pyrrolidone (NMP) and dimethylformamide (DMF). This enables the production of homogeneous thin-films ${ }^{14,21,32,33}$ and as a result of the aforementioned properties the sulfonated melanin is an attractive option for device fabrication. 
(a)<smiles>[R]c1cc2cc(O)c(O)cc2[nH]1</smiles>

(HQ)

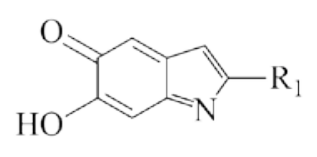

(QI)<smiles>[R]c1cc2cc([O])c(O)cc2[nH]1</smiles>

(SQ) (b)

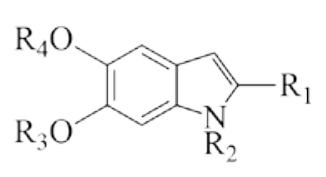

Sulfonated-monomer<smiles>[R]c1cc2c([nH]1)=CC(=O)C(=O)C=2</smiles>

(IQ)<smiles>[R]c1cc2cc(O)c(O)cc2[nH]1</smiles>

$\left(\mathrm{N}-\mathrm{def} \mathrm{f}^{\prime}\right)$

Where: $\mathrm{R}_{1}=\mathbf{H}$ (for DHI species) or $\mathbf{C O O H}$ (for DHICA species)

$\mathrm{R}_{2}=\mathbf{H}$ or $\mathrm{SO}_{2} \mathbf{C H}_{3}$ or $\mathrm{CH}_{3}$

$\mathrm{R}_{3}=\mathbf{H}$ or $\mathbf{S O}_{2} \mathrm{CH}_{3}$

$\mathrm{R}_{4}=\mathbf{H}$ or $\mathrm{SO}_{2} \mathrm{CH}_{3}$

Figure 1. (a) Different monomeric species of standard melanin. (b) A proposed monomeric moiety structure of sulfonated melanin. ${ }^{30}$

Recently, we have reported a successful demonstration of a sulfonated melanin $\mathrm{pH}$ sensor, which showed effective transduction of an ionic activity $(\mathrm{pH})$ into an electronic signal. ${ }^{34}$ However, to date most of the charge transport studies of sulfonated melanin were evaluated through conductivity as a function of temperature ${ }^{21}$ or electrochemical measurements. ${ }^{32}$ Furthermore, what has not been explored is its potential free radical properties, an aspect which is central to the structure-propery relationships in standard non-functionalized melanins. Considering that free-radicals may either be a potential charge carrier or at least be an indirect spin-label for charge transport, ${ }^{35,36}$ a better understanding of the sulfonated mlanin's paramagnetic properties would be a welcome addition to the study of sulfonated melanin's charge transport properties. Hence, in this work, we report new insights into sulfonated melanin's free radical species through hydration-controlled continuous-wave $(\mathrm{CW}) \mathrm{X}$-band electron paramagnetic resonance (EPR) spectroscopy accompanied by electronic structure calculations.

\section{Experimental}

\section{Melanin synthesis}

Four different sulfonated melanin derivatives (S-melanins) were obtained through the oxidation of DL-DOPA (Sigma-Aldrich) in dimethylsulfoxide (DMSO) following previously published procedures. ${ }^{21,29,37}$ Briefly, $1.50 \mathrm{~g}$ of DL-DOPA (Sigma-Aldrich, 98\%) and $0.93 \mathrm{~g}$ of benzoyl peroxide (Vetec, 75.0-80.0 \%) were dissolved in $200 \mathrm{~mL}$ of 
DMSO (PA, Vetec, 99.9\%) and kept under magnetic bar stirring for 58 days at $\pm 27^{\circ} \mathrm{C}$, room temperature (SMel), or for eleven days in a temperature-controlled silicone bath at $100{ }^{\circ} \mathrm{C}$ (SMel-T). The solution was heated at $140{ }^{\circ} \mathrm{C}$ to evaporate $3 / 4$ of the DMSO and then mixed in acetonitrile (Synth, 99.5\%) and stirred for two days. Afterward, the acetonitrile solution was centrifuged at $2500 \mathrm{rpm}$ for 15 minutes and the precipitate extracted was dried at $90^{\circ} \mathrm{C}$ in an oven. Another approach to synthesize S-melanin uses a stainless-steel reactor with a capacity of $150 \mathrm{~mL}$ using a mixture of $60 \mathrm{~mL}$ of DMSO and $0.45 \mathrm{~g}$ of DL-DOPA. This solution was kept stirring for six days with $4 \mathrm{~atm}$ of internal pressure (SMel-4P) or for three days with an internal pressure of 8 atm (SMel-8P). The internal pressure was increased by adding $\mathrm{O}_{2}$ gas into the reactor. The same sequence of solution concentration, dissolution in acetonitrile, centrifugation, and drying was employed for extraction and purification.

All commercially available chemicals were used without further purification.

\section{In-situ hydration-controlled $C W X$-Band EPR measurements}

Following previously published methods, ${ }^{18}$ approximately $150 \mathrm{mg}$ of each Smelanin derivative was crushed to a fine powder and pressed into a pellet of $13 \mathrm{~mm}$ diameter and $1 \mathrm{~mm}$ thickness using a standard pellet press under 10 tons of pressure for 5 minutes. The resulting pellets were then broken into small pieces suitable to fit into a vacuum capable EPR quartz tube (3 mm internal diameter).

Hydration-controlled experiments were performed using a vacuum line setup previously reported ${ }^{18}$ under dry and wet conditions ( $0 \%$ and $80 \%$ relative humidity $(\mathrm{RH})$, respectively) to account for the hygroscopic nature of the melanin materials. ${ }^{38,39}$ The $\mathrm{CW}$ X-band EPR spectra were recorded using a Bruker EMX Micro X, CW-EPR spectrometer equipped with a high power cavity/high-sensitivity ER4119HS resonator. The spectra were taken at a modulation frequency of $100 \mathrm{kHz}$, modulation amplitude of $0.025 \mathrm{mT}$ with a scan width of $6 \mathrm{mT}$ and microwave powers of 0.03 or $10.00 \mathrm{~mW}$. It is important to note here that the diode current, a proxy for the quality factor Q, of the EPR cavity did not change after the addition of water vapor into the system. Such behavior indicates that the variation of the signal intensity is not an experimental artifact caused by dielectric losses, ${ }^{40}$ but rather change in the spin concentration.

\section{Data analysis}


The EPR spectra were analyzed in a similar manner to previously published work. ${ }^{19}$ EasySpin (v.5.2.13) ${ }^{41}$ was used with the pepper routine, using pseudo-Voigtian ( 65\% Lorentzian character) function and isotropic broadening. The proposed pseudo-Voigt lineshape is a requirement for a complete analysis of the EPR signal asymmetry and is discussed in more detail later. ${ }^{42}$ To reduce the number of variables used in the fittings, part of the parameters associated with the g-values, linewidths and lineshapes were kept fixed during the fitting procedure. However, it should be noted that in the initial simulations, we have considered different amounts of resonant lines and the spectral parameters converged to values close to those discussed below. OriginPro 9.0 was used to doubly integrate the first derivative EPR spectra to determine spin concentrations. In this work, we confine our analysis to low microwave power spectra only to avoid any possible saturated effects originating from a higher microwave intensity.

\section{Electronic structure calculations}

To better understand the origin of the EPR signal in S-melanins, electronic structure calculations were conducted for charged and radicalar species of sulfonated DHI and DHICA units. Figure 2 illustrates the evaluated structures which were designed based on the common building units of standard and non-functionalized melanins (NFmelanins). ${ }^{6,35}$ 
<smiles>[R]Oc1cc2cc([R])[nH]c2cc1O[R]</smiles><smiles>[R]OC1=CC2=NC([R])=CC2=CC1=O</smiles><smiles>[R]Oc1cc2[nH]c([R])cc2cc1[O]</smiles><smiles>[R]Oc1cc2cc([R])[nH]c2cc1O</smiles><smiles>[R]Oc1cc2c(cc1O[R])[NH2+]C([R])=C2</smiles><smiles>[R]Oc1cc2cc([R])n([R])c2cc1O[R]</smiles><smiles>[R]c1cc2c(n1[R])=CC(=O)C(=O)C=2</smiles><smiles>[R]Oc1cc2c(cc1OC)cc([R])n2[R]</smiles><smiles>[R]Oc1cc2cc([R])n([R])c2cc1O</smiles><smiles></smiles><smiles></smiles><smiles>[R]c1cc2c(n1C)=CC(=O)C(=O)C=2</smiles><smiles>[R]Oc1cc2c(cc1O[2H])cc([R])n2C</smiles><smiles>[R]Oc1cc2cc([R])n(C)c2cc1[O-]</smiles>

where:

$\mathrm{R}_{\mathrm{s}}=\mathrm{SO}_{2} \mathrm{CH}_{3}$ $\mathrm{R}=\mathrm{COOH}$ or $\mathrm{H}$

Figure 2. S-melanin units considered in the DFT calculations.

50 distinct conformers were designed for each monomeric structure via molecular dynamic (MD) calculations at high temperatures. For this purpose, the monomeric structures were placed in contact with a thermal reservoir at $1000 \mathrm{~K}$ and allowed to evolve (during 500 ps); a distinct conformer structure was stored for every 10 ps for subsequent geometry optimization. ${ }^{35,43}$

All the obtained conformers were pre-optimized in a Hartree-Fock (HF) approach using the PM6 semi-empirical approximation as implemented in the MOPAC2016 computational package. ${ }^{44,45}$ The structures were then fully optimized in vacuo in the framework of the density functional theory (DFT) using the B3LYP ${ }^{46-49}$ hybrid exchangecorrelation functional and 6-31G basis set on all the atoms. The most stable neutral structures were selected and fully optimized in a DFT/B3LYP/6-31G(d,p) approach. These structures were then used for subsequent evaluations. DFT-based geometry optimizations were carried out with the aid of GAMESS (DFT/B3LYP/6-31G approach for pre-optimization for all the conformers) and Gaussian 09 (DFT/B3LYP/6-31G(d,p) for the most stable structures) computational packages. ${ }^{50,51}$ 
In addition to sulfonated semiquinone radicals (S-SQ-based structures), charged species were also evaluated to identify possible paramagnetic centers in S-melanin. In these cases, the addition (anion) or removal (cation) of one electron in the structures give rise to systems with doublet spin configurations that are sensitive EPR spectroscopy. ${ }^{52,53}$ The charged units were then re-optimized with a DFT/B3LYP/6-31G(d,p) approach, by considering the neutral optimized structures as starting geometries. For S-Ndef ${ }^{+}$, only its anionic structure (i.e., S-Ndef ${ }^{+}+\mathrm{e}^{-}=$S-Ndef) was considered.

The g-factors ( $g_{\text {iso }}$ ) were obtained from the average of the principal values of the $g^{T} g$ matrix ( $g_{x x}, g_{y y}$ and $g_{z z}$, square roots of the eigenvalues of $\left.g^{T} g\right)^{52}$ estimated with the aid of Orca 3.0.3 computational package, ${ }^{54}$ using the DFT/B3LYP/6-31G(d,p) approach.

For the optimization and evaluation of non-functionalized units (NF-melanins), the same approach was employed as described above, but with the most stable geometries reported in ref ${ }^{35}$ as the starting structures.

The energies of the frontier levels of the building blocks (the Highest Occupied Molecular Orbital, Eномо; and the Lowest Unoccupied Molecular Orbital, ELUMo) were estimated from the ionization potentials (IP) and electron affinities (EA) as follows:

$$
\begin{aligned}
& \square_{\square \square \square \square}=-\square \square=-\left(\square_{\square}\left(\square^{+}\right)-\square_{\square}(\square)\right) \\
& \square_{\square \square \square \square}=-\square \square=-\left(\square_{\square}(\square)-\square_{\square}\left(\square^{-}\right)\right)
\end{aligned}
$$

where $E_{T}\left(M^{+}\right), E_{T}(M)$ and $E_{T}\left(M^{-}\right)$represent the total energy of the molecule $M$ at distinct charge configurations (cation, neutral and anion), considering the same geometry (optimized for $\mathrm{M}$ at neutral configuration).

The potentialities of charge transfer between S and NF-melanin building blocks were estimated via donor-acceptor map (DAM) based on each molecule's philicities ( $\square^{+}$ and $\square^{-}$):

$$
\begin{aligned}
& \square_{\square}^{-}=\frac{(3 \square \square+\square \square)^{2}}{16(\square \square-\square \square)} \\
& \square_{\square}^{+}=\frac{(\square \square+3 \square \square)^{2}}{16(\square \square-\square \square)},
\end{aligned}
$$

and relative electron-accepting $\left(\mathrm{R}_{\mathrm{a}}\right)$ and electron-donating $\left(\mathrm{R}_{\mathrm{d}}\right)$ powers, given by: ${ }^{55-57}$

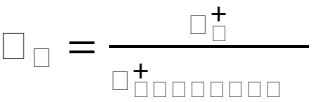

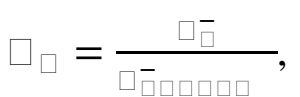

where $\square_{\mathrm{M}^{-}}$and $\square_{\mathrm{M}}{ }^{+}$represent the philicity powers of the molecule $\mathrm{M}$, and $\square^{+}$Fluorine and $\square$-sodium the powers associated with fluorine and sodium atoms (estimated from 
experimental IP and EA values ${ }^{56}$ ), that are considered electron acceptor and electron donating model systems. ${ }^{56}$

Low (higher) values of $\square^{-}\left(\square^{+}\right)$imply a higher capacity for donating (accepting) electrons. In this sense, $R_{a}$ and $R_{d}$ descriptors indicate the charge transfer potentiality of $M$ in relation to fluorine and sodium atoms. $R_{a}>1$ and $R_{d}<1$ indicate that $M$ is a more effective electron acceptor and electron donor than the model systems.

\section{Results and Discussion}

Considering that the hydration level is a key parameter for the paramagnetic, electronic characterization of melanin, we systematically studied the free radical EPR signal of S-melanin derivatives at different hydration levels (dry, $0 \% \mathrm{RH}$, and wet, $80 \%$ $\mathrm{RH}$ ). As displayed in Figure 3 (solid lines, $0.03 \mathrm{~mW}$ ), no significant spectral feature or intensity differences were observed between the hydration levels (also See Figure S1 in Supplementary Material). The asymmetric spectra obtained showed an isotropic g value ranging from 2.0044 to 2.0047 (Figure S2).
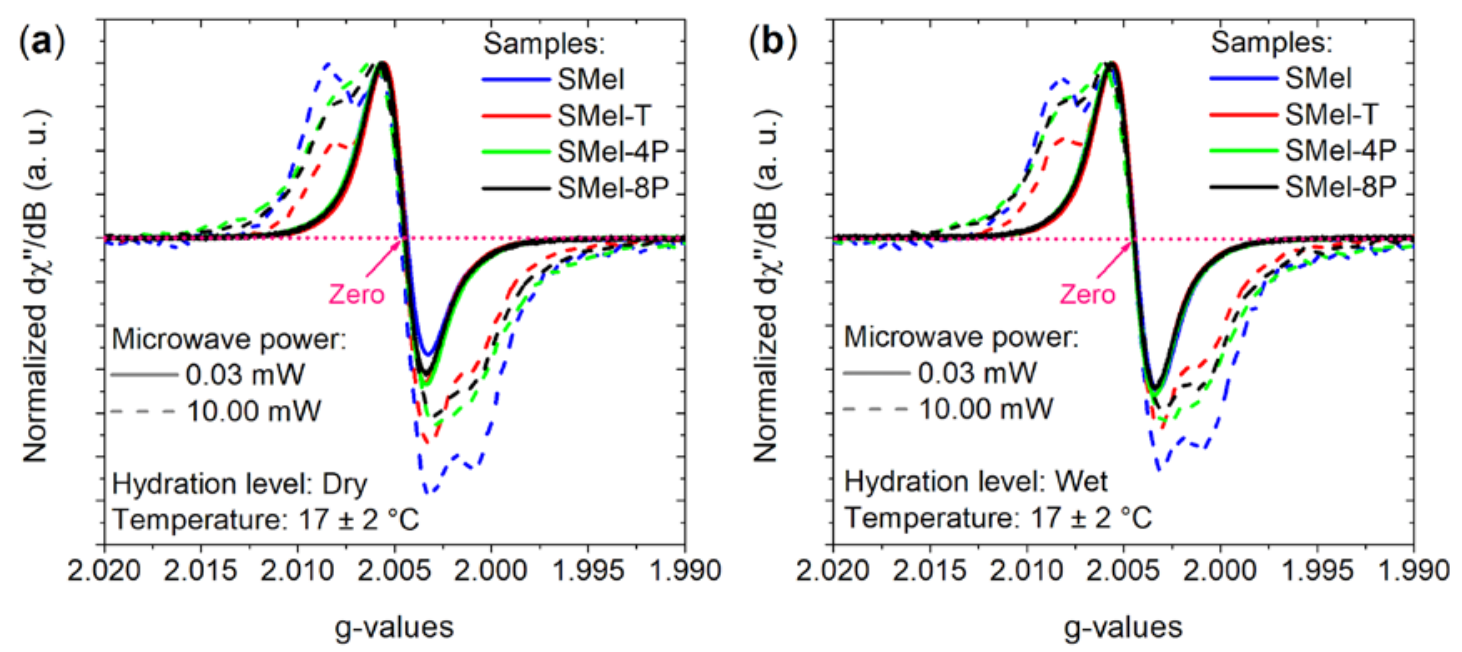

Figure 3. CW X-band EPR spectra normalized to the positive peak for the four S-melanin under (a) dry $(0 \% \mathrm{RH})$ or (b) wet $(80 \% \mathrm{RH})$ hydration conditions, at low and high microwave power (see legend).

It is known that the EPR spectra of melanins can change dramatically with the application of higher microwave powers. As a matter of fact, the resultant EPR spectra 
recorded at $10.00 \mathrm{~mW}$ (dashed lines in Figure 3) show that the EPR spectra exhibit new features from broader lines, which are not present at the low microwave power. Such behavior could be related to distorted lineshapes as a result of saturation transfer phenomena between different components that may be present in the system. ${ }^{52,53}$ However, it has also been shown that changing the nature of the paramagnetic species, due to changing the $\mathrm{pH}$, can modify the EPR spectrum in the same way as saturation effects. ${ }^{18,36,58}$ Hence, an alternative possibility to the high microwave power behavior suggests that the paramagnetic system of S-melanin could be composed of at least two different radical populations with similar g-values that have slightly different spin-lattice relaxation rates, in a similar manner to NF-melanin (see Figure S3 for comparison).

Another interesting feature in Figure 3 is the change in lineshape; that is, the signal asymmetry around the maximum absorption (or when the derivative lines cross zero). Such behavior would be an indicator that the different lines that result in the EPR signal (i.e., different paramagnetic species) do not have the same g-value, or, the species have a different anisotropic g.

To gain more insights into the nature of the paramagnetic properties, the low microwave power spectra were analyzed using simulations with EasySpin. ${ }^{41}$ It should be stressed that even though the EPR signal may be anisotropic in nature, we restricted our analysis to consider only isotropic g-values to not over parameterize the problem. This assumption provides an excellent fit. However, if some species have a g anisotropy, a complete analysis will only be possible at high-field EPR spectroscopy, ${ }^{59}$ which will be explored in future work.

Initially, our simulations considered the existence of one or two resonant lines. However, the fits were poor. Therefore, a third component was introduced. Figure 4 shows experimental spectra (black), the simulated fitted line (red) and the three simulated components (blue, pink and green) for SMel-T, which is used as an example (see Figure S5-S7 for the other samples). The g-values and linewidths obtained for the three different lines in the dry samples were kept fixed and used for the wet spectra simulations (Figure S4 and Figure S5-S7 for the other samples) to facilitate the data analysis. The only free variables were the intensities of the signals, which were transformed into an estimated spin concentration of each species (Figure 5). Reasonable fits with a root-mean-square deviation (RMSD) below 0.020 were obtained (compared to 0.060 for one or 0.050 for two species). Hence, given the modeling parameters, the simulations potentially indicate the presence of at least three paramagnetic centers in S-melanin. 


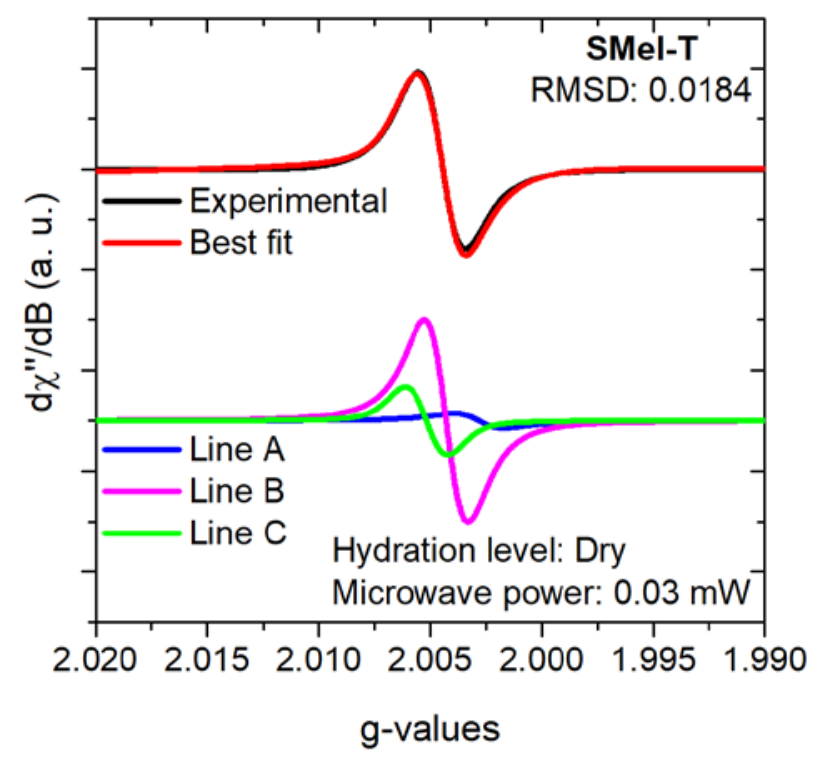

Figure 4. SMel-T CW X-band EPR spectra simulations at a microwave power of 0.03 $\mathrm{mW}$ under dry condition ( $0 \% \mathrm{RH})$. Black and red lines represent the experimental and simulated spectra, while blue, pink and green the three components used in the fitting. SMel-T simulations for wet spectra are shown in Figure S4, whereas for the other samples, please see Figures S5-S7. The key differences in the parameters are shown in Figure 5.
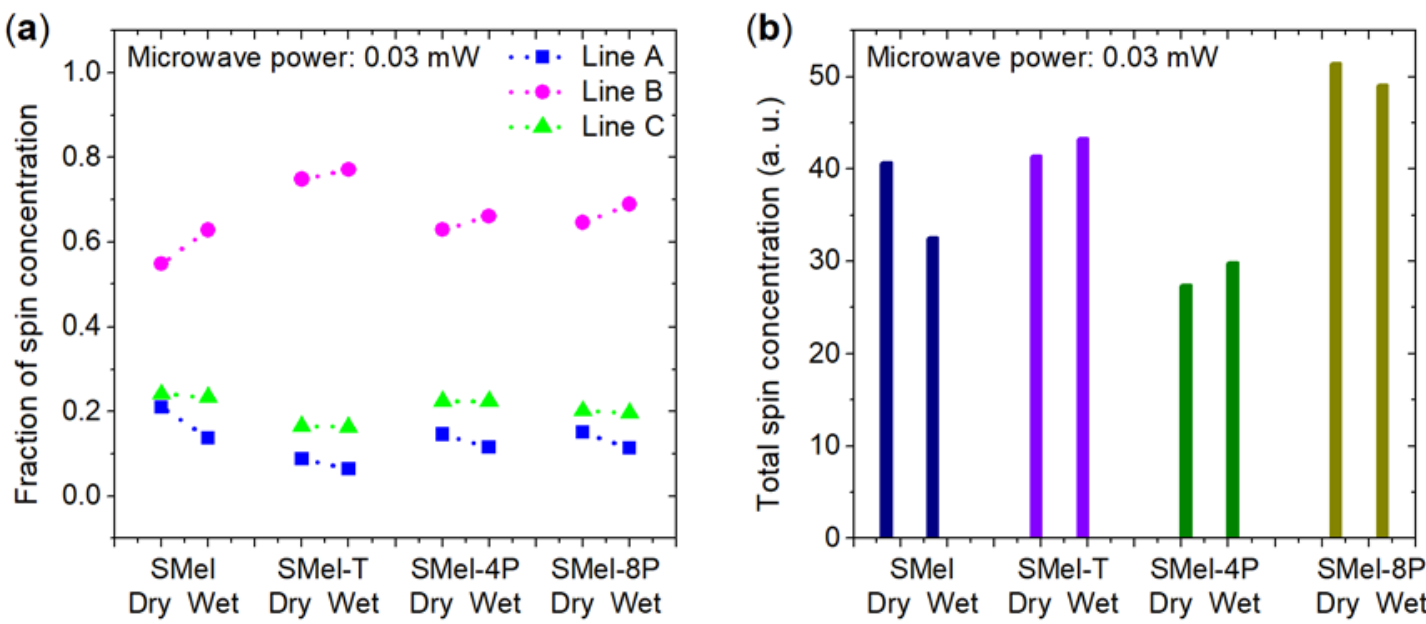

Figure 5. Spin concentration for each component of S-melanin at a microwave power of $0.03 \mathrm{~mW}$. The uncertainty bars (propagated from the difference between modeled and experimental data) are smaller than the data point resolution. 
To understand the data in Figure 5, we refer to Table 1 where the the estimated spectroscopic parameters for each component are shown.

Table 1. Spectral parameters of the simulated signals for all four S-melanin samples.

\begin{tabular}{ccc}
\hline Resonant lines & g-Values & $\Delta H_{\mathbf{P P}}(\mathbf{m T})$ \\
\hline A & 2.0028 & 0.456 \\
\hline B & 2.0043 & 0.421 \\
\hline C & 2.0052 & 0.392 \\
\hline
\end{tabular}

Based on the spectroscopic parameters in Table 1, it is possible to see that the gvalue of components A, B and C are compatible with NF-melanin (Figure S8) and they are within experimental variations to previous simulations. ${ }^{19}$ The similarity is an indication that the S-melanin paramagnetic response could be consistent with NFmelanin. In fact, given the relative concentration of sulfur to nitrogen atoms as estimated via XPS reported elsewhere, ${ }^{29}$ NF-melanin moieties should be present within the polymer.

Figure 5 indicates that the paramagnetic species exhibited a hydration dependence, which is in agreement with NF-melanin data, but far more subdued in intensity (compare with Figure S9-S10). Usually, the NF-melanin free-radical system is discussed in terms of a supposed carbon-centered radical (CCR) and semiquinone free-radical (SFR). It was proposed that CCR has g values below 2.004 and it is located within the stacked oligomeric sheets. In contrast, the SFR is situated at the exterior of the macro-structure, in close contact with the environment, with g higher than 2.004. ${ }^{18,35}$ An interesting feature about these two groups is that experiments carried out with hydration-controlled atmosphere have shown that water and $\mathrm{OH}^{-}$ions can quench the CCR signals generating SFR due to destacking of NF-oligomers. ${ }^{18}$ Hence, considering that the paramagnetic centers of NF-melanin are integrally involved in its electrochemical processes, it should follow that similar free-radicals are present in S-melanin because their electrochemical properties seem to be virtually the same. ${ }^{32,34}$ Therefore, after the addition of water in the system, the intensity of component $\mathbf{A}$ decreases and component $\mathbf{B}$ increases (Figure 5a), which could potentially indicate that a CCR species is destroyed to form SFR. 
On the other hand, component $\mathbf{C}$ has lower (to none) hydration effects on the spin concentration. Assuming that the small linewidth of component $\mathbf{C}$ indicates that it could be an SFR species (similarly to NF-melanin), ${ }^{35}$ the result could potentially mean an absence of surface interactions between S and NF-moieties and water. Considering that S-melanin may be more hydrophobic, due to the presence of additional methyl groups attached to the sulfonates, and its solubility in DMSO, it is likely that the macromolecule that was formed under the synthesis conditions have led to S-structure sitting on the surface of the particle. Consequently, S-moieties could i) be not affected by hydration (due to the mentioned hydrophobic properties); and, ii) act as effective spacers between NF-units to prevent the interaction of NF-monomers with the hydrating environment.

Figure 5 also shows that the hydration sensitivity of SMel, regarding the spin concentration, is higher than the other sulfonated samples, which could point towards different macro-structure sizes. If one assumes that SMel has smaller particles compared to SMel-T (as shown by TEM, see Figure S11), it would be expected that the water molecules can easily penetrate through its macro-structure, providing a more effective route to quench the CCR species. However, a natural question is whether the paramagnetic centers in S-melanin are indeed NF-melanin moieties, somehow trapped and isolated even after water addition, or whether the lineshapes can potentially be ascribed to concomitant S-melanin radicals with similar EPR response.

We also note that from the results presented above indicates that the different polymerization processes ${ }^{29}$ of S-melanin did not affect its paramagnetic properties and the EPR signal can be fitted with the same components. Consequently, the monomeric nature of these samples seems to be more important than the secondary structure, in accordance with a previous theoretical study. ${ }^{35}$

To investigate the above concerns and investigate if one could tell the difference between the two potential scenarios in more detail, we turn to computational modeling. As suggested above, the monomeric structures seem to play a significant role in the EPR signal. Therefore, to evaluate which paramagnetic center could be associated with the three simulated species, DFT calculations were conducted for charged and neutral radical species of S-melanin units. Figure 6 shows the distribution of g-values obtained. $\mathbf{0 1}$ and 02 numbers refer to Sulfonated-DHI and Sulfonated-DHICA units, respectively. Color maps of the spin density are presented in the Supplementary Material (Figures S12-S15). 


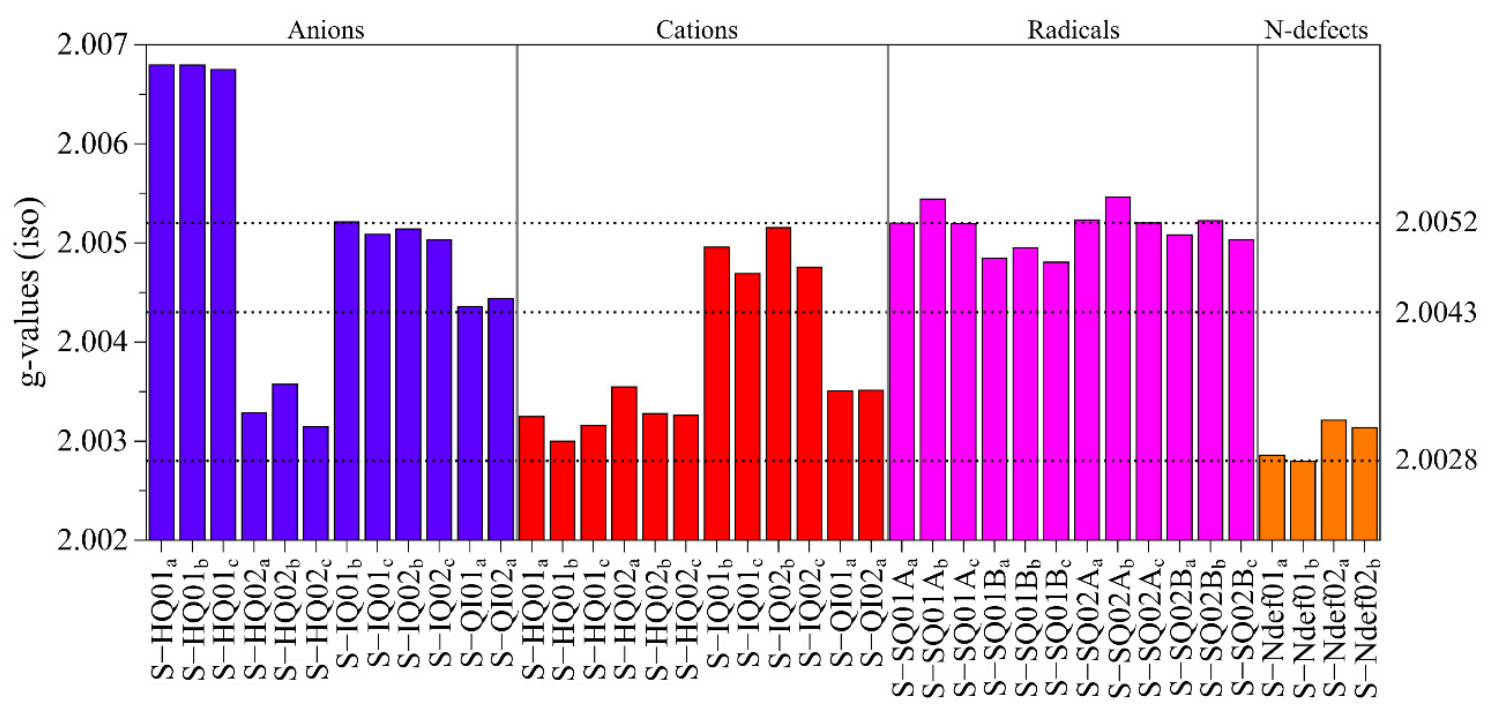

Figure 6. Distribution of g-values obtained for charged (anion and cation) and radical units of S-melanin with unpaired spins (i.e., EPR active species). The left side legend indicates the g-values scale, whereas, on the right side, the g-values estimated via spectral simulation (Table 1).

According to Figure 6, there is a wide distribution of g-values for S-melanin with similar values within different sulfonated structures. The range of g-values agrees with those reported for NF-melanin. ${ }^{35}$

Generally, the results from the modeling in Figure 6 indicates that the nature of the substituent attached to the nitrogen does not play a significant role in the g-values of the compounds. In a like manner, for most of the units, the presence of $\mathrm{COOH}$ groups in the 02 structures does not lead to significant effects in the g-values, with a relevant exception for S-HQ (anion) units. A detailed analysis of S-HQ01 (anion) structure shows that the inclusion of one electron in the neutral (pre-optimized) molecule leads to the releasing of one $-\mathrm{SO}_{2} \mathrm{CH}_{3}$ group attached to the quinone oxygen, with the spin localized on the - $\mathrm{SO}_{2} \mathrm{CH}_{3}$ radical (see Figure S11). Such an effect is not observed in the S-HQ02 counterpart, which promotes a more homogeneous electronic distribution over the molecule. This result indicates that the higher g-values could be associated with trapped sulfonated groups $\left(-\mathrm{SO}_{2} \mathrm{CH}_{3}\right)$ coming from the S-melanin synthesis that deserves more in-depth analyses. Additional calculations conducted for a hypothetical $\bullet \mathrm{SO}_{2} \mathrm{CH}_{3}$ radical (using the same theoretical approaches in the methods) leads to a g-value of 2.0059, which is slightly higher than the signals $\mathbf{C}$ presented in Table 1 and are compatible with those reported elsewhere. ${ }^{60}$ 
The g-value of S-units can be divided in three main groups, which are associated with $\mathrm{g}<2.0040$ and $\mathrm{g}>2.0040$ paramagnetic centers:

- g<2.0040: S-HQ02 (anion), S-HQ01 (cation), S-HQ02 (cation), S-QI01 (cation); S-QI02 (cation); S-Ndef01 and S-Ndef02.

- $\quad$ > 2.0040: S-IQ01 (anion), S-IQ02 (anion), S-QI01 (anion), S-QI02 (anion), SIQ01 (cation), S-IQ02(cation), S-SQA01; S-SQB01; S-SQ02A and S-SQ02B.

- Interlayer sulfonated groups: S-HQ01 (anion).

These subdivisions are compatible with those reported for NF-melanins, ${ }^{35}$ with sulfonated and non-functionalized units being classified in the same category. The only exceptions are the degraded S-HQ01-based structures, mentioned before. Such a result indicates that S-melanin units share similar paramagnetic variables to NF-melanins units. In particular, the results suggest that the resonant line $\mathbf{A}$ could be related to low g-value structures like S-HQ02 (anion), S-HQ (cation) and S-Ndef, while line B can be ascribed to S-QI (anion) species with intermediate g-values. S-SQ (radicals) and S-IQ (anion) species are candidates for line $\mathbf{C}$.

To better interpret the electrochemistry associated with the formation of paramagnetic centers in S-melanins, the relative alignment between the frontier energy levels (HOMO and LUMO, Figure 7) and the electron donation/acceptance powers of the S-units were estimated and compared with NF-monomers (Figure 8).

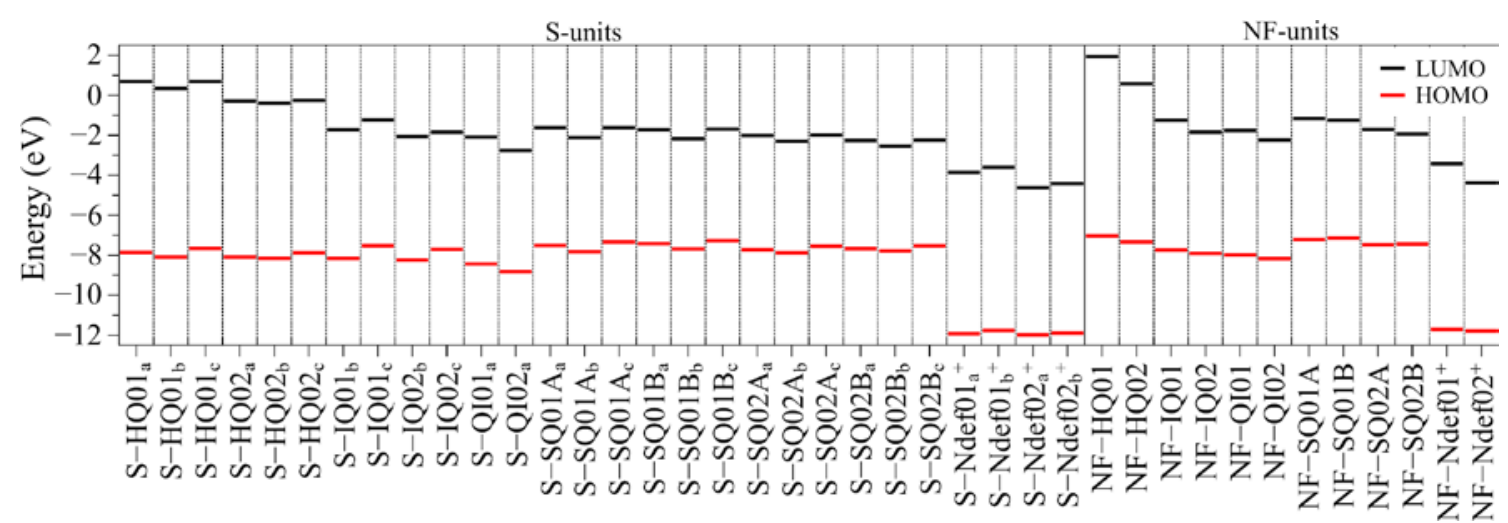

Figure 7. Frontier energy levels of neutral S- and NF-melanin building blocks. 


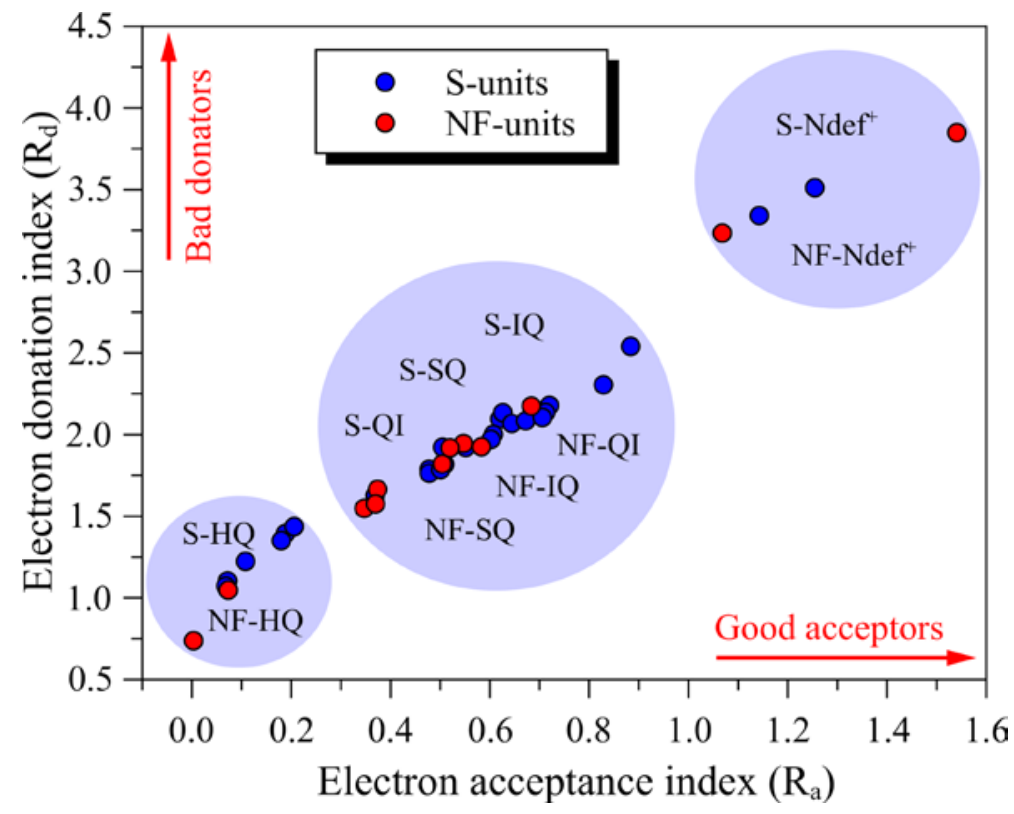

Figure 8. DAM of S and NF-melanin monomers.

S and NF-units present similar electronic features. A detailed analysis exhibit that NF-monomers show a slightly higher tendency to donate electrons than their Scounterparts. In contrast, the opposite behavior is obtained for S-monomers, i.e., Smonomers tends to accept electrons more easily. However, these differences appear to be slight and may indicate similar electrochemistry, which may explain the similarity between S-melanin and NF-melanin electrochemical data. ${ }^{32,34}$ A relevant feature to note is the definition of $\mathrm{Ndef}^{+}$as effective electron acceptors, which indicate the plausibility of the formation of Ndef-based paramagnetic species in the system.

As mentioned above, we anticipate that a number of the molecular units present in S-melanin do not contain sulfonate groups. Hence, the formation of paramagnetic centers in S-melanins could include charge transfer and/or trapping between $\mathrm{S}$ and NFmonomers, as well as to the surrounding environment, leading to the formation of anionic and cationic structures that can contribute to the EPR signal. The similarity between the frontier levels of NF and S-units and the presence of trapping states associated with $\mathrm{Ndef}^{+}$ is compatible with this hypothesis.

The following could be the case: i) the resonant line $\mathbf{A}$ can be attributed to the neutralized S-Ndef species that act as electron traps in the systems (as well as other cationic species generated in the process); ii) the resonant line $\mathbf{B}$ can be ascribed to S-QI (anion) which is reinforced by the low LUMO levels of these structures; iii) the resonant line $\mathbf{C}$ is mainly associated with S-SQ, S-IQ (anion) and S-IQ (cation) species. The 
proposed structures are consistent with the previous discussion regarding the water effect on the spin concentration. For example, the disruption of the duplet configuration of SNdef centers (S-Ndef $+\mathrm{H}_{2} \mathrm{O}=\mathrm{S}-\mathrm{HQ}+\mathrm{H}_{3} \mathrm{O}^{+}+\mathrm{e}^{-}$) decreases component $\mathbf{A}$ after hydration (Figure 5a). With the rearranging of the neutral S-Ndef center, there will be a change in the electronic charge experienced in the system, and, consequently, the extra electron would be donated to S-QI units originating anionic S-QI (component B) or to S-HQ units originating anionic S-HQ and $\bullet \mathrm{SO}_{2} \mathrm{CH}_{3}$ radical. Conisdering the simulated parameters (Table 1) and the energy levels of the monomeric structures (Figure 7), the first case seems to be the most probable one. Note that a possible transformation of component $\mathbf{A}$ into component $\mathbf{B}$, while maintaining component $\mathbf{C}$ unchanged, could result in the apparent lack of hydration effect shown in Figure S2.

The electronic conductivity behavior of NF-melanin is usually viewed with the presence of NF-Ndef been able to trap electrons and/or holes. At the same time, the formation of plausible NF-IQ, NF-QI and NF-SQ species can be a source of electron and proton. ${ }^{15,35,61}$ Therefore, the similarities between the free radical nature and frontier energy levels of NF and S-melanin would point to similar electronic properties. However, it is possible to speculate that S-melanin should present lower protonic current and less sensitivity to hydration due to the presence of S-groups. The possibility of S-melanin derivatives could be an electronic dominated system, vs. the NF-melanin, which is protonic dominated, though sharing similarities in charge transport properties opens additional opportunities for device applications.

\section{Conclusion}

In this report, we have combined hydration-controlled, solid-state X-band CW EPR spectroscopy and electronic structure calculations to study the nature of paramagnetic species of S-melanin derivatives. Our results show that the S-melanin EPR signal appears similar to NF-melanin. Simulation analyses of the EPR signals suggest that the S-melanin signal is composed of three different resonant lines that are compatible with NF-melanin. Intriguingly, the theoretical calculations indicate that the EPR signal could arise from distinct S-units, in particular S-Ndef, S-QI (anion) and S-SQ (radical)/S-IQ (anion). The similarities between the free radical nature of NF and S-melanin provided some insight into the structure and the potential charge transport behavior of the later. 


\section{Supplementary material}

Synthetic procedure for standard and non-functionalized melanin derivatives; additional EPR parameters and simulations, spin densities and transmission microscopy images.

\section{Acknowledgment}

JVP, ABN and CFOG gratefully acknowledge the financial support of São Paulo Research Foundation (FAPESP; grants 2012/03116-7, 2013/07296-2, 2015/23000-1, 2018/02411-1), Brazilian Coordination for the Improvement of Higher Education Personnel (CAPES), Brazilian National Council for Scientific and Technological Development (CNPq, grants: 573636/2008-7, 308360/2018-4, 420449/2018-3). The research was also supported by resources supplied by the Center for Scientific Computing (NCC/GridUNESP) of São Paulo State University (UNESP). A.B.M. is a Sêr Cymru II fellow and the results incorporated in this work have received funding from the European Union's Horizon 2020 research and innovation program under the Marie SkłodowskaCurie grant agreement No 663830. P.M. is a Sêr Cymru Research Chair funded by the Welsh European Funding Office (European Regional Development Fund) through the Sêr Cymru II Program and an Honorary Professor at the University of Queensland.

\section{References}

${ }^{1}$ R.M. Owens and G.G. Malliaras, MRS Bull. 35, 449 (2010).

${ }^{2}$ S. Inal, G.G. Malliaras, and J. Rivnay, Nat Commun 8, 1767 (2017).

${ }^{3}$ S. Kim, B. Keisham, and V. Berry, Materials Today Nano 9, 100063 (2020).

${ }^{4}$ P. Meredith, C.J. Bettinger, M. Irimia-Vladu, A.B. Mostert, and P.E. Schwenn, Rep. Prog. Phys. 76, 034501 (2013).

${ }^{5}$ N. Amdursky, E.D. Głowacki, and P. Meredith, Adv. Mater. 31, 1802221 (2019).

${ }^{6}$ P. Meredith and T. Sarna, Pigment Cell Research 19, 572 (2006).

${ }^{7}$ M. d'Ischia, A. Napolitano, A. Pezzella, P. Meredith, and M. Buehler, Angew. Chem. Int. Ed. (2020).

${ }^{8}$ L. Hong and J.D. Simon, J. Phys. Chem. B 111, 7938 (2007).

${ }^{9}$ L. Panzella, G. Gentile, G. D’Errico, N.F. Della Vecchia, M.E. Errico, A. Napolitano, C. Carfagna, and M. d'Ischia, Angewandte Chemie International Edition 52, 12684 (2013).

${ }^{10}$ S. Gidanian and P.J. Farmer, Journal of Inorganic Biochemistry 89, 54 (2002).

${ }^{11}$ C.-L. Serpentini, C. Gauchet, D. de Montauzon, M. Comtat, J. Ginestar, and N. Paillous, Electrochimica Acta 45, 1663 (2000).

12 J.Y. Lin and D.E. Fisher, Nature 445, 843 (2007).

${ }^{13}$ P. Meredith, B.J. Powell, J. Riesz, S.P. Nighswander-Rempel, M.R. Pederson, and E.G. Moore, Soft Matter 2, 37 (2006).

${ }^{14}$ J. Wünsche, F. Cicoira, C.F.O. Graeff, and C. Santato, Journal of Materials Chemistry B 1, 3836 (2013).

${ }^{15}$ A.B. Mostert, B.J. Powell, F.L. Pratt, G.R. Hanson, T. Sarna, I.R. Gentle, and P. Meredith, 
Proceedings of the National Academy of Sciences 109, 8943 (2012).

${ }^{16}$ J. Wünsche, Y. Deng, P. Kumar, E. Di Mauro, E. Josberger, J. Sayago, A. Pezzella, F. Soavi, F. Cicoira, M. Rolandi, and C. Santato, Chem. Mater. 27, 436 (2015).

${ }^{17}$ K.A. Motovilov, V. Grinenko, M. Savinov, Z.V. Gagkaeva, L.S. Kadyrov, A.A. Pronin, Z.V. Bedran, E.S. Zhukova, A.B. Mostert, and B.P. Gorshunov, RSC Adv. 9, 3857 (2019).

${ }^{18}$ A.B. Mostert, G.R. Hanson, T. Sarna, I.R. Gentle, B.J. Powell, and P. Meredith, The Journal of Physical Chemistry B 117, 4965 (2013).

19 J.V. Paulin, A. Batagin-Neto, and C.F.O. Graeff, J. Phys. Chem. B 123, 1248 (2019).

${ }^{20}$ M.S. Blois, A.B. Zahlan, and J.E. Maling, Biophysical Journal 4, 471 (1964).

${ }^{21}$ S.N. Dezidério, C.A. Brunello, M.I.N. da Silva, M.A. Cotta, and C.F.O. Graeff, Journal of NonCrystalline Solids 338-340, 634 (2004).

${ }^{22}$ K.J. Lawrie, P. Meredith, and R.P. McGeary, Photochem. Photobiol. 84, 632 (2008).

${ }^{23}$ A. Pezzella, A. Iadonisi, S. Valerio, L. Panzella, A. Napolitano, M. Adinolfi, and M. d'Ischia, J. Am. Chem. Soc. 131, 15270 (2009).

${ }^{24}$ M. d'Ischia, K. Wakamatsu, A. Napolitano, S. Briganti, J.-C. Garcia-Borron, D. Kovacs, P. Meredith, A. Pezzella, M. Picardo, T. Sarna, J.D. Simon, and S. Ito, Pigment Cell Melanoma Res 26, 616 (2013).

${ }^{25}$ S.R. Cicco, M. Ambrico, P.F. Ambrico, M.M. Talamo, A. Cardone, T. Ligonzo, R. Di Mundo, C. Giannini, T. Sibillano, G.M. Farinola, P. Manini, A. Napolitano, V. Criscuolo, and M. d'Ischia, J. Mater. Chem. C 3, 2810 (2015).

${ }^{26}$ E.S. Bronze-Uhle, J.V. Paulin, M. Piacenti-Silva, C. Battocchio, M.L.M. Rocco, and C.F. de O. Graeff, Polymer International 65, 1339 (2016).

${ }^{27}$ M. Iacomino, J. Mancebo-Aracil, M. Guardingo, R. Martín, G. D’Errico, M. Perfetti, P. Manini, O. Crescenzi, F. Busqué, A. Napolitano, M. d’Ischia, J. Sedó, and D. Ruiz-Molina, IJMS 18, 2169 (2017).

${ }^{28}$ R. Micillo, M. lacomino, M. Perfetti, L. Panzella, K. Koike, G. D'Errico, M. d'Ischia, and A. Napolitano, Pigment Cell Melanoma Res. 31, 475 (2018).

${ }^{29}$ J.V. Paulin, A.G. Veiga, Y. Garcia-Basabe, M.L.M. Rocco, and C.F. Graeff, Polymer International 67, 550 (2018).

${ }^{30}$ E.S. Bronze-Uhle, A. Batagin-Neto, P.H.P. Xavier, N.I. Fernandes, E.R. de Azevedo, and C.F.O. Graeff, Journal of Molecular Structure 1047, 102 (2013).

${ }^{31}$ M. Piacenti-Silva, A.A. Matos, J.V. Paulin, R.A. da S. Alavarce, R.C. de Oliveira, and C.F. Graeff, Polym. Int. 65, 1347 (2016).

${ }^{32}$ L.G. Albano, E. Di Mauro, P. Kumar, F. Cicoira, C.F. Graeff, and C. Santato, Polym. Int. 65, 1315 (2016).

${ }^{33}$ M.I.N. da Silva, S.N. Dezidério, J.C. Gonzalez, C.F.O. Graeff, and M.A. Cotta, J. Appl. Phys. 96, 5803 (2004).

${ }^{34}$ Z. Tehrani, S.P. Whelan, A.B. Mostert, J.V. Paulin, M.M. Ali, E.D. Ahmadi, C.F.O. Graeff, O.J. Guy, and D.T. Gethin, 2D Mater. 7, 024008 (2020).

${ }^{35}$ A. Batagin-Neto, E.S. Bronze-Uhle, and C.F. de O. Graeff, Physical Chemistry Chemical Physics 17, 7264 (2015).

${ }^{36}$ A.B. Mostert, S.B. Rienecker, C. Noble, G.R. Hanson, and P. Meredith, Science Advances 4, eaaq1293 (2018).

${ }^{37}$ M. Piacenti-Silva, E.S. Bronze-Uhle, J.V. Paulin, and C.F.O. Graeff, Journal of Molecular Structure 1056-1057, 135 (2014).

${ }^{38}$ A.B. Mostert, K.J.P. Davy, J.L. Ruggles, B.J. Powell, I.R. Gentle, and P. Meredith, Langmuir 26, 412 (2010).

${ }^{39}$ A.J. Clulow, A.B. Mostert, M. Sheliakina, A. Nelson, N. Booth, P.L. Burn, I.R. Gentle, and P. Meredith, Soft Matter 13, 3954 (2017).

${ }^{40}$ D.P. Dalal, S.S. Eaton, and G.R. Eaton, Journal of Magnetic Resonance (1969) 44, 415 (1981).

${ }^{41}$ S. Stoll and A. Schweiger, Journal of Magnetic Resonance 178, 42 (2006).

${ }^{42}$ F.J. Grady and D.C. Borg, J. Am. Chem. Soc. 90, 2949 (1968). 
${ }^{43}$ A. Batagin-Neto, E.F. Oliveira, C.F.O. Graeff, and F.C. Lavarda, Molecular Simulation 39, 309 (2013).

${ }^{44}$ J.J.P. Stewart, J. Comput.-Aided Mol. Des. 4, 1 (1990).

45 J.J.P. Stewart, MOPAC2016 (Stewart Computational Chemistry, Colorado Springs, CO, USA, 2018).

${ }^{46}$ A.D. Becke, The Journal of Chemical Physics 98, 5648 (1993).

${ }^{47}$ C. Lee, W. Yang, and R.G. Parr, Physical Review B 37, 785 (1988).

${ }^{48}$ S.H. Vosko, L. Wilk, and M. Nusair, Canadian Journal of Physics 58, 1200 (1980).

${ }^{49}$ P.J. Stephens, F.J. Devlin, C.F. Chabalowski, and M.J. Frisch, The Journal of Physical Chemistry 98, 11623 (1994).

${ }^{50}$ M. J. Frisch, G. W. Trucks, H. B Schlegel, G. E Scuseria, M. Robb, J. Cheeseman, G. Scalmani, V. Barone, B. Mennucci, G. A. H. Petersson, H. Nakatsuji, M. Caricato, X. Li, H. P Hratchian, A. F Izmaylov, J. Bloino, G. Zheng, J. L Sonnenberg, M. Hada, and D. Fox, (2009).

${ }^{51}$ M.W. Schmidt, K.K. Baldridge, J.A. Boatz, S.T. Elbert, M.S. Gordon, J.H. Jensen, S. Koseki, N. Matsunaga, K.A. Nguyen, S. Su, T.L. Windus, M. Dupuis, and J.A. Montgomery, Journal of Computational Chemistry 14, 1347 (1993).

${ }^{52}$ J.A. Weil and J.R. Bolton, Electron Paramagnetic Resonance: Elementary Theory and Practical Applications, 2nd ed. (Wiley, New York, 2006).

${ }^{53}$ C.P. Poole, Electron Spin Resonance: A Comprehensive Treatise on Experimental Techniques (John Wiley \& Sons, New York, 1983).

${ }^{54}$ F. Neese, Wiley Interdisciplinary Reviews: Computational Molecular Science 2, 73 (2012).

55 J.L. Gázquez, A. Cedillo, and A. Vela, The Journal of Physical Chemistry A 111, 1966 (2007).

${ }^{56}$ A. Martínez, M.A. Rodríguez-Gironés, A. Barbosa, and M. Costas, The Journal of Physical Chemistry A 112, 9037 (2008).

${ }^{57}$ D.A. Hernandez and F.J. Tenorio, Structural Chemistry 29, 741 (2018).

${ }^{58}$ S.B. Rienecker, A.B. Mostert, G. Schenk, G.R. Hanson, and P. Meredith, J. Phys. Chem. B 119, 14994 (2015).

${ }^{59}$ K. Möbius and A. Savitsky, High-Field EPR Spectroscopy on Proteins and Their Model Systems: Characterization of Transient Paramagnetic States (Royal Society of Chemistry, Cambridge, UK, 2009).

${ }^{60}$ M. Geoffroy and E.A.C. Lucken, The Journal of Chemical Physics 55, 2719 (1971).

${ }^{61}$ G.G.B. Alves, F.C. Lavarda, C.F.O. Graeff, and A. Batagin-Neto, Journal of Molecular Graphics and Modelling 98, 107609 (2020). 


\section{Supplementary material}

\section{Shedding light on the free radical nature of sulfonated melanins}

J.V. Paulin ${ }^{\mathrm{a}, \mathrm{b}}$, A. Batagin-Neto ${ }^{\mathrm{a}, \mathrm{c}}$, E. Richards ${ }^{\mathrm{d}}$, P. Meredith ${ }^{\text {b,g }}$, C.F.O. Graeff ${ }^{\mathrm{a}, \mathrm{e}}$, A.B. Mostert ${ }^{\mathrm{f}}$

a São Paulo State University (UNESP), School of Sciences, Postgraduate Program in Materials Science and Technology (POSMAT), Bauru, Brazil.

b Swansea University, Department of Physics, Singleton Park, Swansea, SA2 8PP, United Kingdom.

c São Paulo State University (UNESP), Campus of Itapeva, Itapeva, Brazil

${ }^{\mathrm{d}}$ Cardiff University, School of Chemistry, Cardiff, United Kingdom.

e São Paulo State University (UNESP), School of Sciences, Department of Physics, Bauru, Brazil.

f Swansea University, Department of Chemistry, Singleton Park, Swansea, SA2 8PP, United Kingdom.

g School of Mathematics and Physics, University of Queensland, St Lucia Campus, Brisbane Queensland 4072, Australia.

Corresponding authors:

a.batagin@unesp.br (A. Batagin-Neto)

a.b.mostert@swansea.ac.uk (A.B.Mostert) 


\section{Non-functionalized melanin synthesis:}

Non-functionalized and insoluble melanin (NFMel-I) was synthesized following the literature procedure: ${ }^{\mathrm{R} 1} 5 \mathrm{~g}$ of DL-DOPA was dissolved in $2 \mathrm{~L}$ of deionized water whose $\mathrm{pH}$ was increased to 8 by adding ammonia (Sigma-Aldrich, 28 \%) and kept under constant stirred with air bubbling for 3 days. Then, concentrated hydrochloric acid (Sigma-Aldrich, 25\%) was used to adjust to $\mathrm{pH} 2$ and precipitate the melanin. A black powder was obtained by filtration and washing several times the aggregated solution.

Soluble NF-melanin derivatives were obtained based on literature: ${ }^{\mathrm{R} 2} 0.3 \mathrm{~g}$ of DLDOPA was dissolved in $60 \mathrm{~mL}$ of MiliQ water $(18 \mathrm{M} \Omega \mathrm{cm})$ and $400 \mu \mathrm{L}$ of ammonium hydroxide (Synth, 28-30\%) added to increase the $\mathrm{pH}$ to 8-9. This mixture was stirred and oxygenated with an air pump for three days (NFMel-S) or in a $150 \mathrm{~mL}$ stainless steel reactor with 6 atm internal pressure of industrial oxygen gas for 6 hours (NFMel-6P) in room temperature $\left( \pm 27^{\circ} \mathrm{C}\right)$. Afterward, the solution filtered using a $3500 \mathrm{MWCO}$ dialysis membrane for four days. The dialysate medium was MiliQ water and it was changed every day. Black powder was obtained after drying the suspended colloidal particles for two days at $90^{\circ} \mathrm{C}$ in an oven.

These different samples have identical structural, but with different degrees of oxidation (NFMel-I $<$ NFMel-S $<$ NFMel-6P).

\section{$X$-Band EPR data}


g-Factor

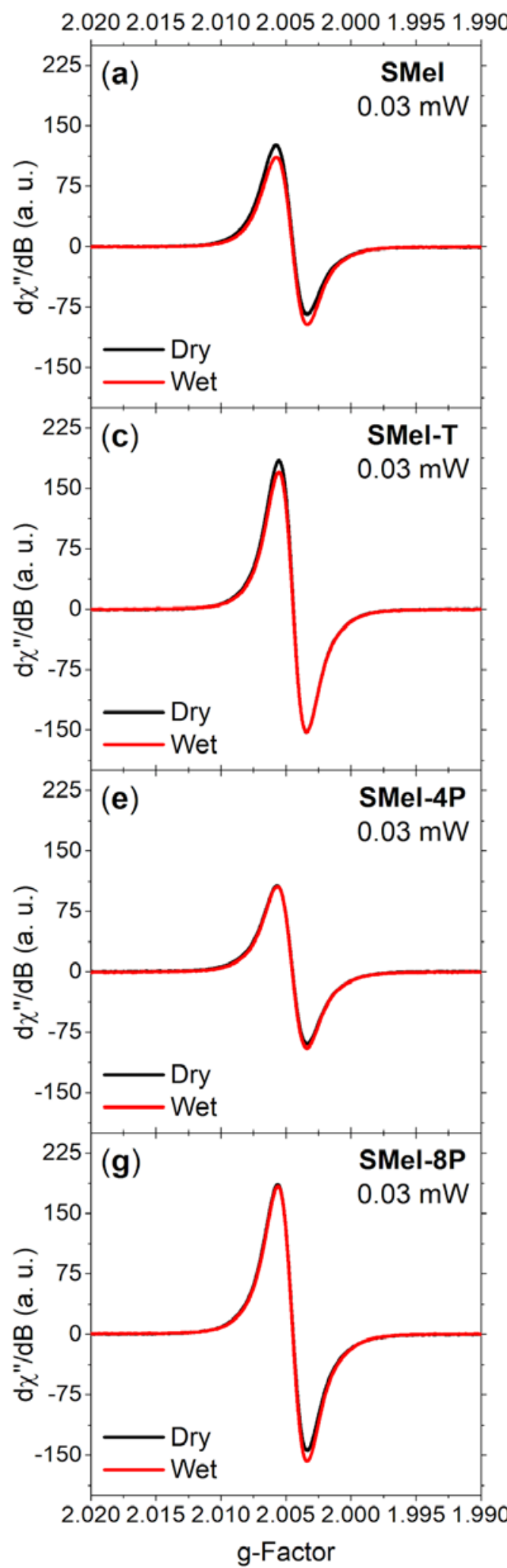

g-Factor

2.0202 .0152 .0102 .0052 .0001 .9951 .990
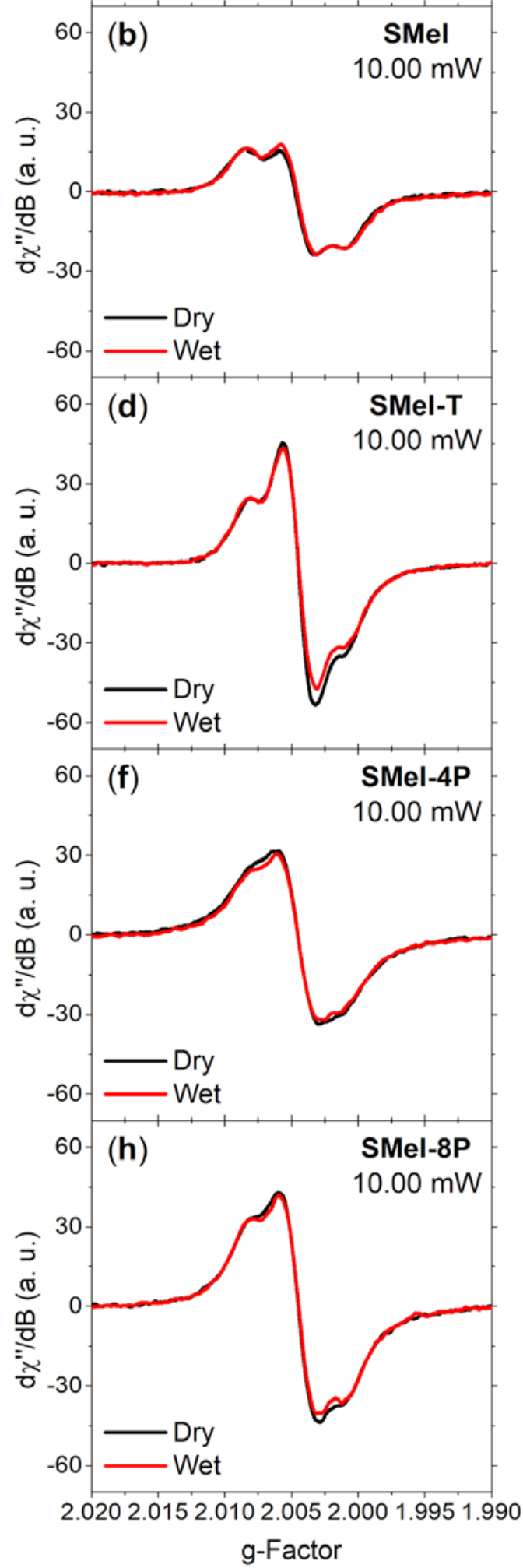

Figure S1. CW X-band EPR spectra for S-melanin under dry and wet hydration level at low (left column) and high (right column) microwave power. 
Table S1. Variation in the spin concentration after the introduction of water in the system. The values present in this table were estimated from the double integration of the EPR signal present in Figure S1. However, note that such values could be a consequence of mathematical artifacts coming from spectral variations in peak asymmetry and linewidth after the hydration (see Figure S2).

\begin{tabular}{ccc}
\hline & \multicolumn{2}{c}{ Spin concentration changes with hydration (\%) } \\
\cline { 2 - 3 } S-melanin samples & $\mathbf{0 . 0 3} \mathbf{~} \mathbf{W}$ & $\mathbf{1 0 . 0 0} \mathbf{~} \mathbf{W}$ \\
\hline SMel & -20.3 & +0.0 \\
\hline SMel-T & +4.7 & -0.3 \\
\hline SMel-4P & +9.4 & -9.6 \\
\hline SMel-8P & -4.7 & -2.9 \\
\hline NFMel-I & -44.7 & -39.4 \\
\hline NFMel-S & -37.7 & -38.5 \\
\hline NFMel-6P & -32.2 & -39.0 \\
\hline
\end{tabular}



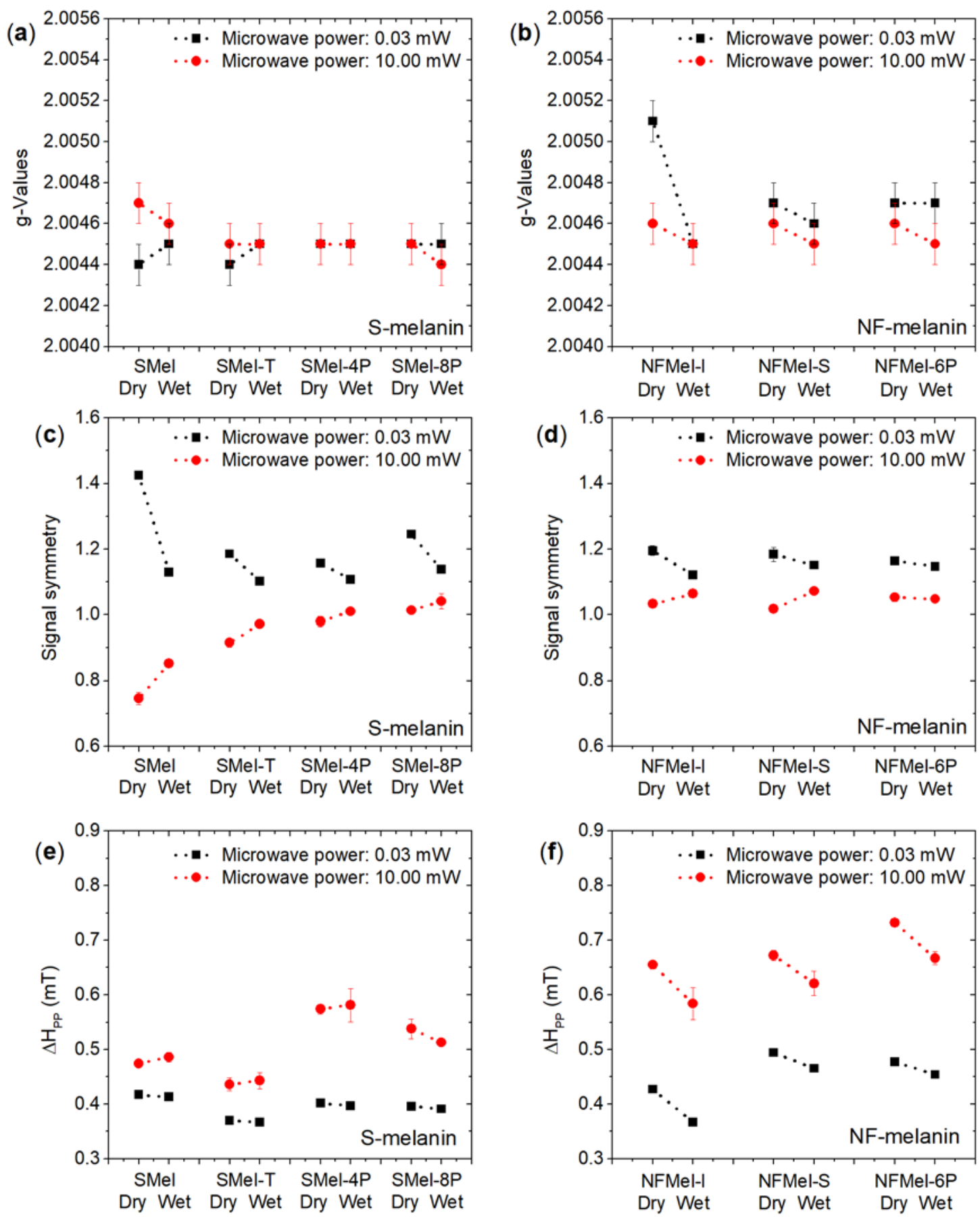

Figure S2. (a, b) g-Values calculated with the fundamental equation of EPR spectroscopy ( $h \square=g \square B_{0}$ ). The deviations were obtained by choosing three different baselines from the positive and negative part of the spectra to get different zero position, that is, magnetic resonance fields $\left(B_{0}\right)$. (c, d) EPR signal symmetry obtained from the ratio between positive and negative intensities. The closer the ratio is to 1 , the more symmetrical the signal. (e, f) The linewidth of the EPR signal. The uncertainty bars, when not shown, are smaller than the data point resolution. 

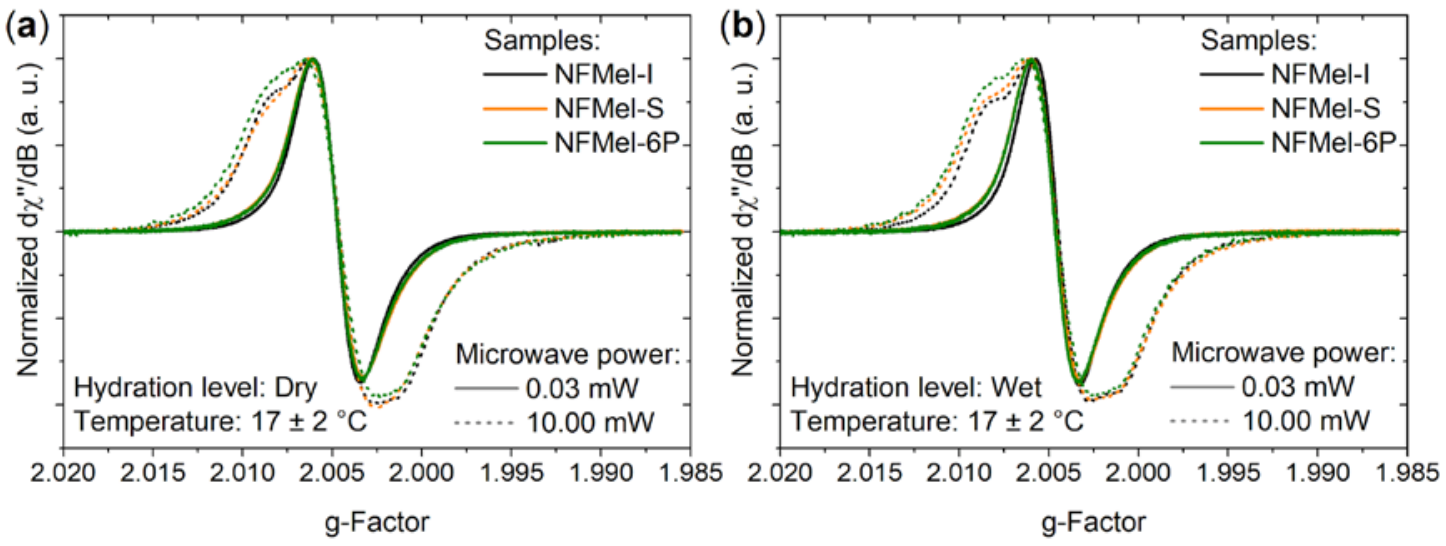

Figure S3. X-band EPR signal of NF-melanin.

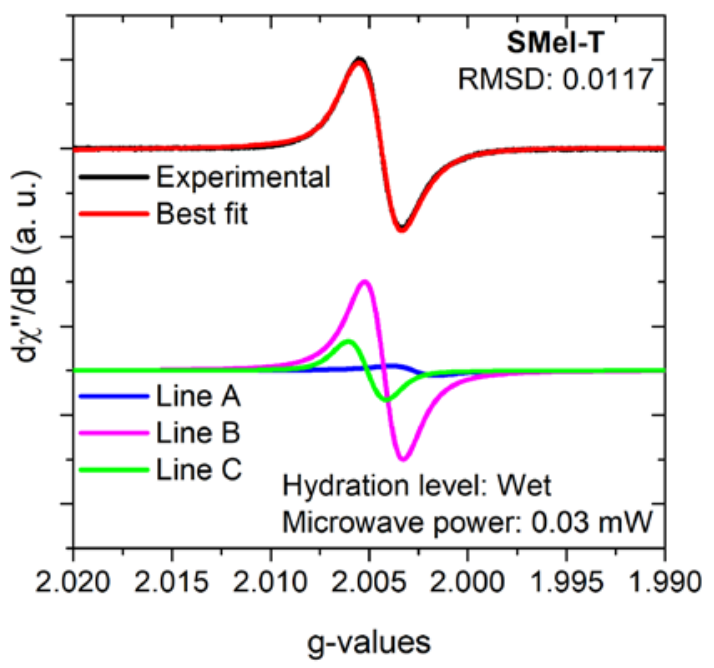

Figure S4. EPR simulation of SMel-T in wet condition (about $80 \%$ humidity).
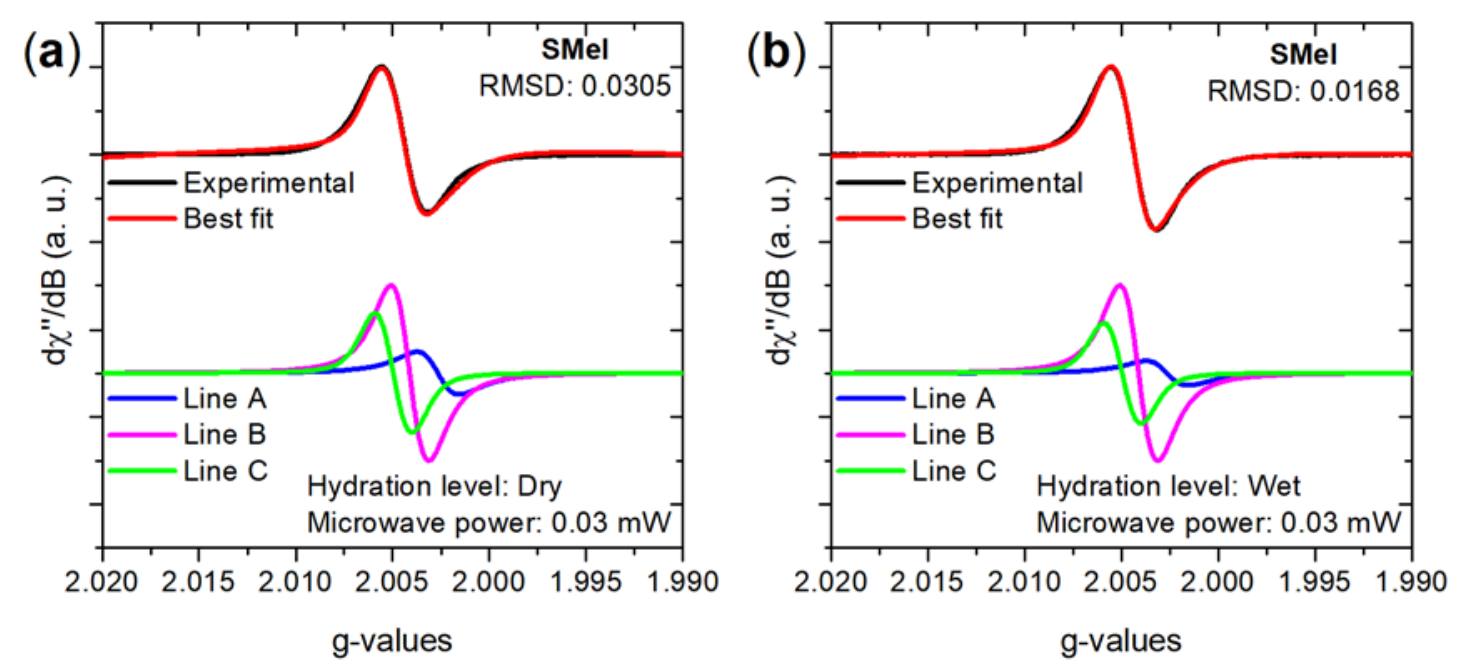

Figure S5. EPR simulation for SMel. 

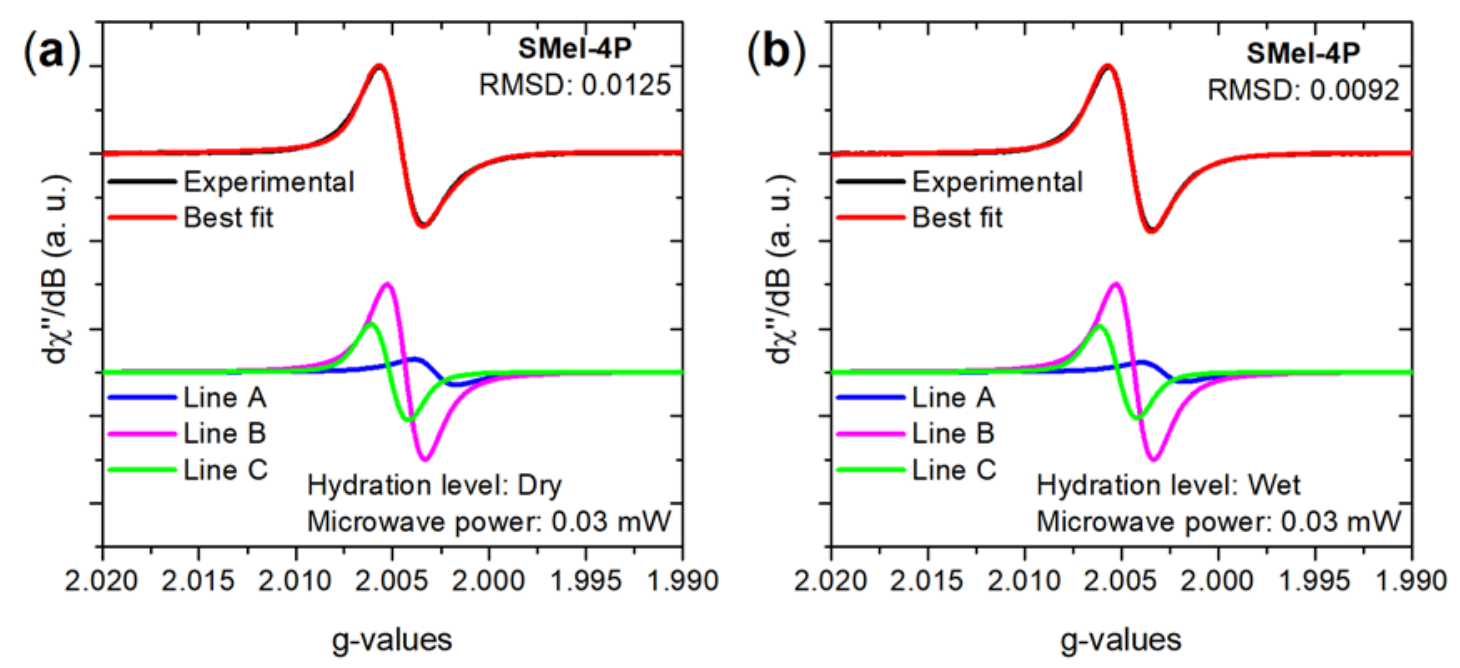

Figure S6. EPR simulation for SMel-4P.
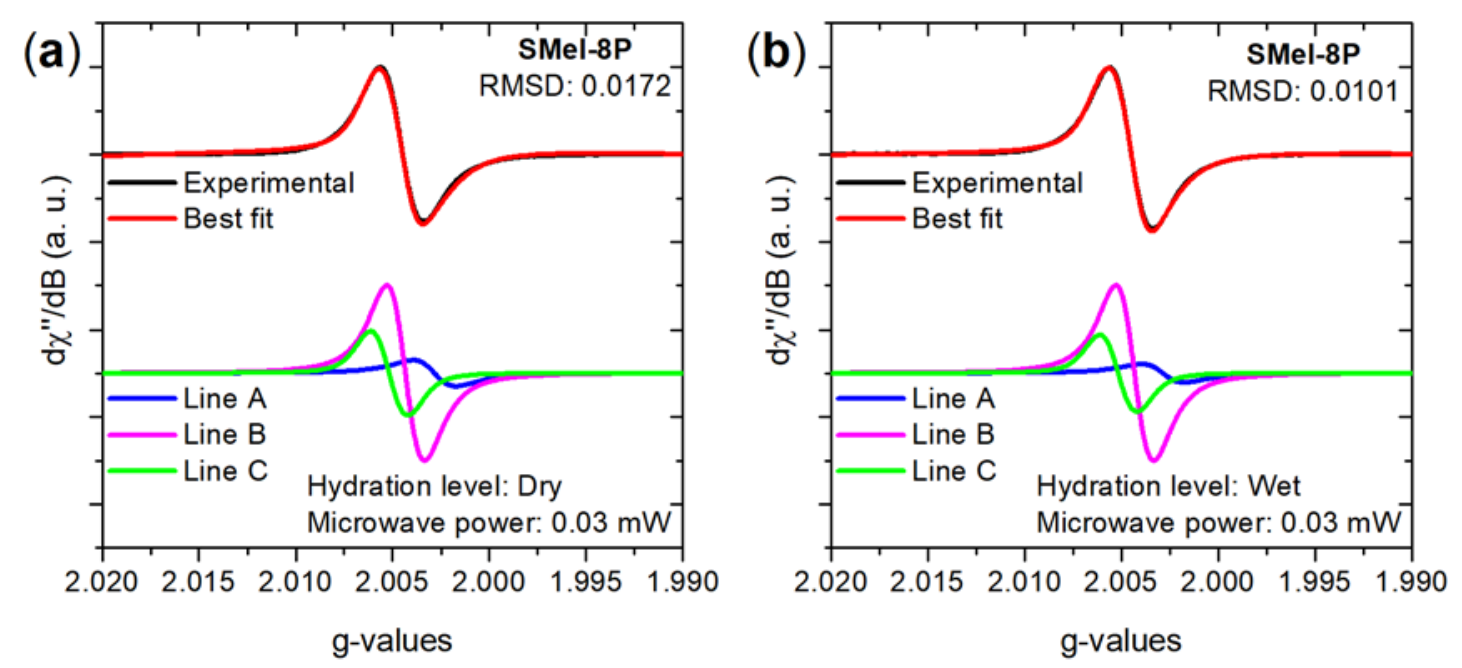

Figure S7. EPR simulation for SMel-8P. 

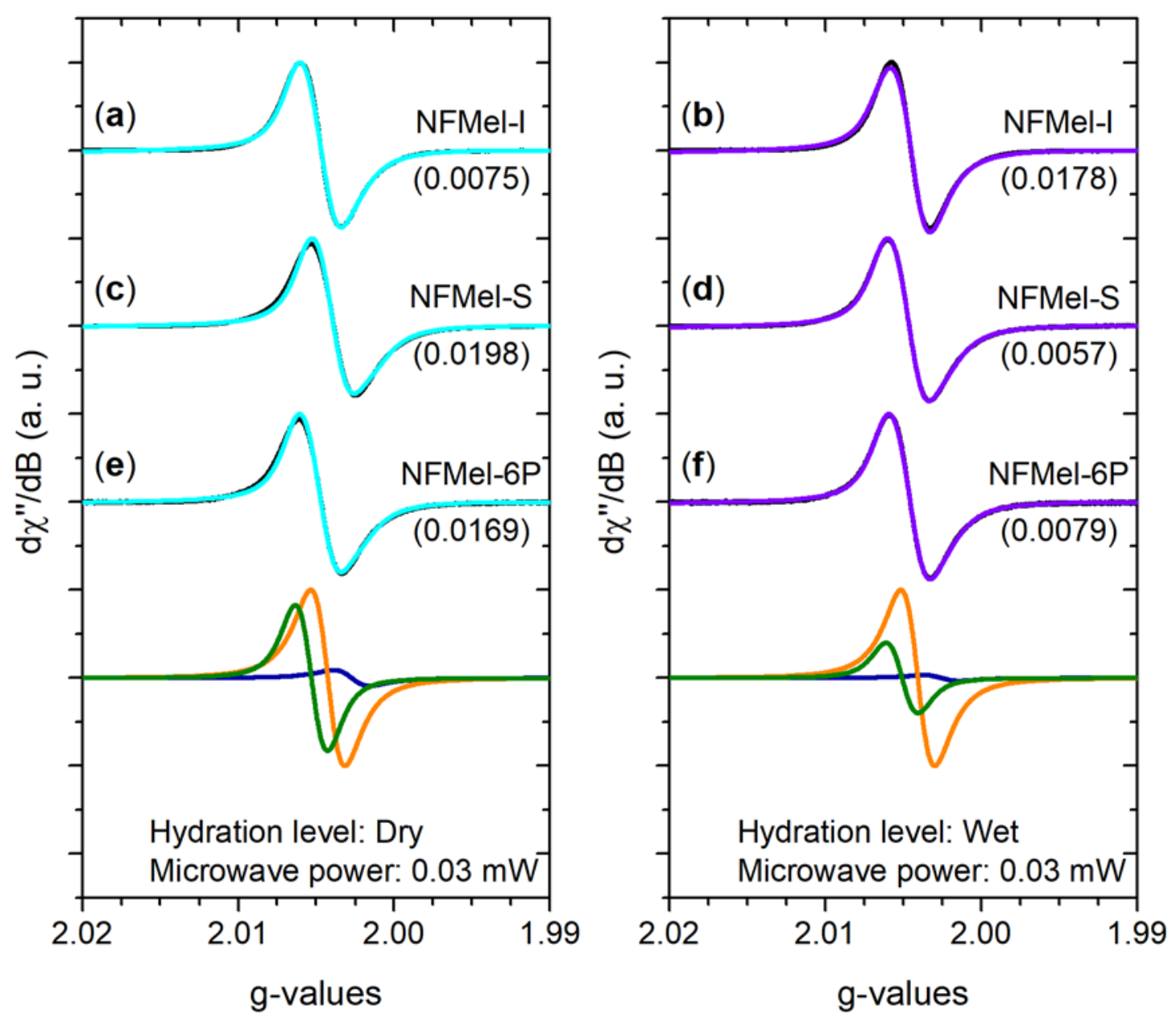

Figure S8. A estimation of the EPR simulation for NFMel-I (a, b), NFMel-S (c, d) and NFMel-6P (e, f) under dry (left) and wet (right) hydration level. The black lines represent the experimental spectra and cyan and violet lines the simulated one at dry and wet hydration level, respectively. Royal-orange-olive lines are the three components used in the fit. The simulated parameters related to g-value and linewidth are shown in Table S2, whereas the different intensities in Figure S9. The numbers between the brackets indicate the RMSD values obtained for each simulation. 
Table S2. Spectral parameters of the simulated signals for all three NF-melanin samples at microwave power of $0.03 \mathrm{~mW}$.

\begin{tabular}{ccc}
\hline Lines & g-Values & $\Delta \mathrm{H}_{\mathbf{P P}(\mathbf{m T})}$ \\
\hline A & 2.0027 & 0.457 \\
\hline B & 2.0043 & 0.468 \\
\hline C & 2.0053 & 0.438 \\
\hline
\end{tabular}
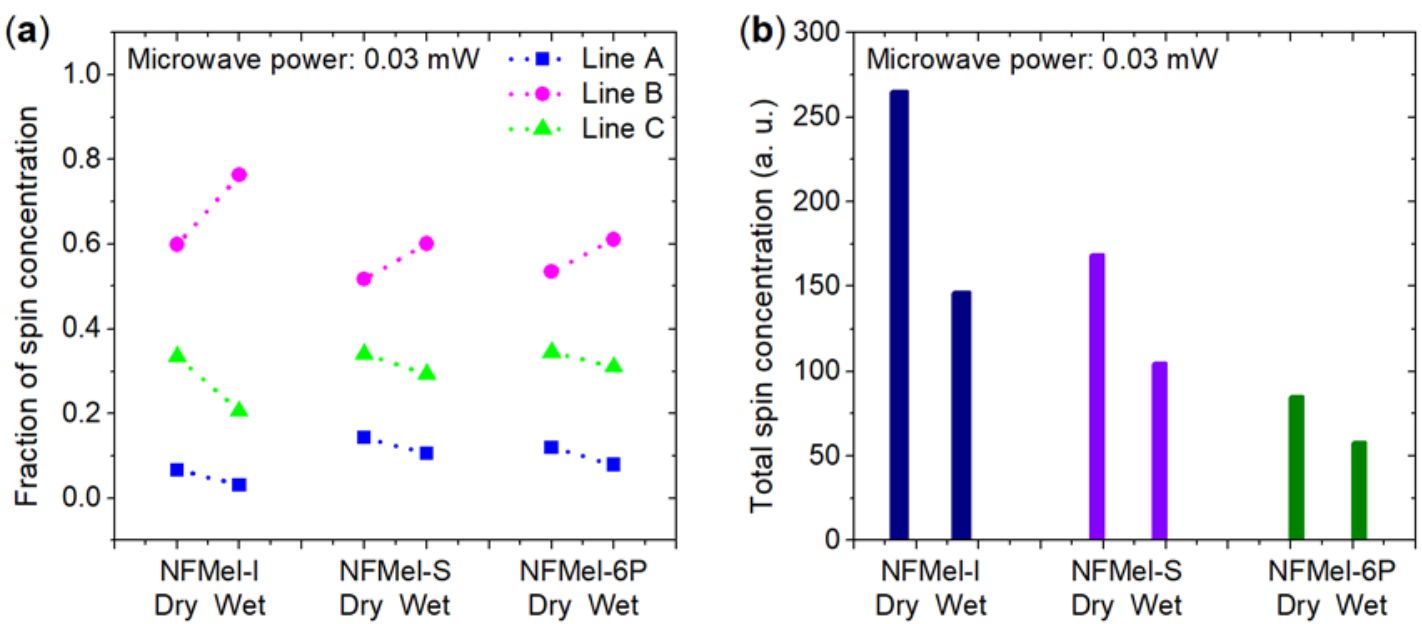

Figure S9. Estimation of the spin concentration of each component of the NF-melanin. The uncertainty bars are smaller than the data point resolution. The decrease in intensity of Line $\mathrm{C}$ is related to the diminished of the linewidth spectra after the addition of water (see Figure S2f and Figure S10). Such behavior is unexpected and is not fully understood as yet. 
g-Factor

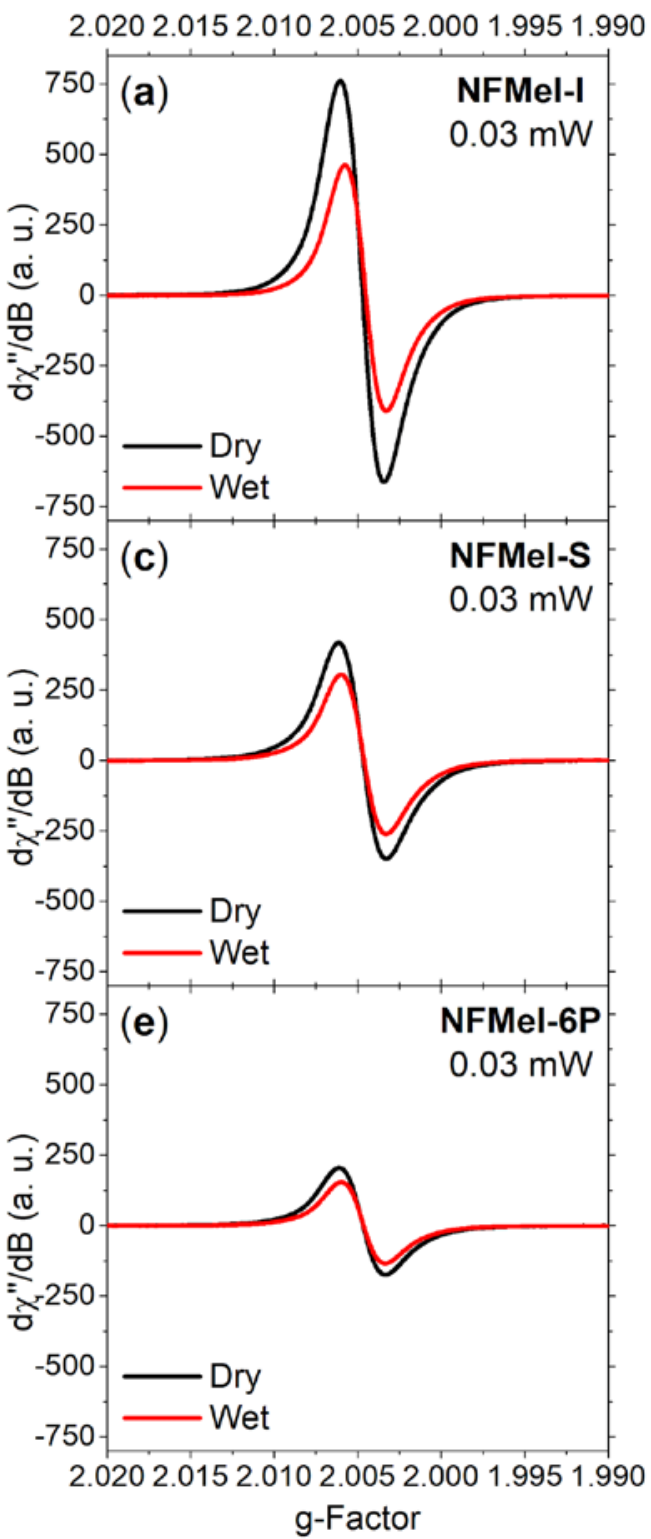

g-Factor

2.0202 .0152 .0102 .0052 .0001 .9951 .990

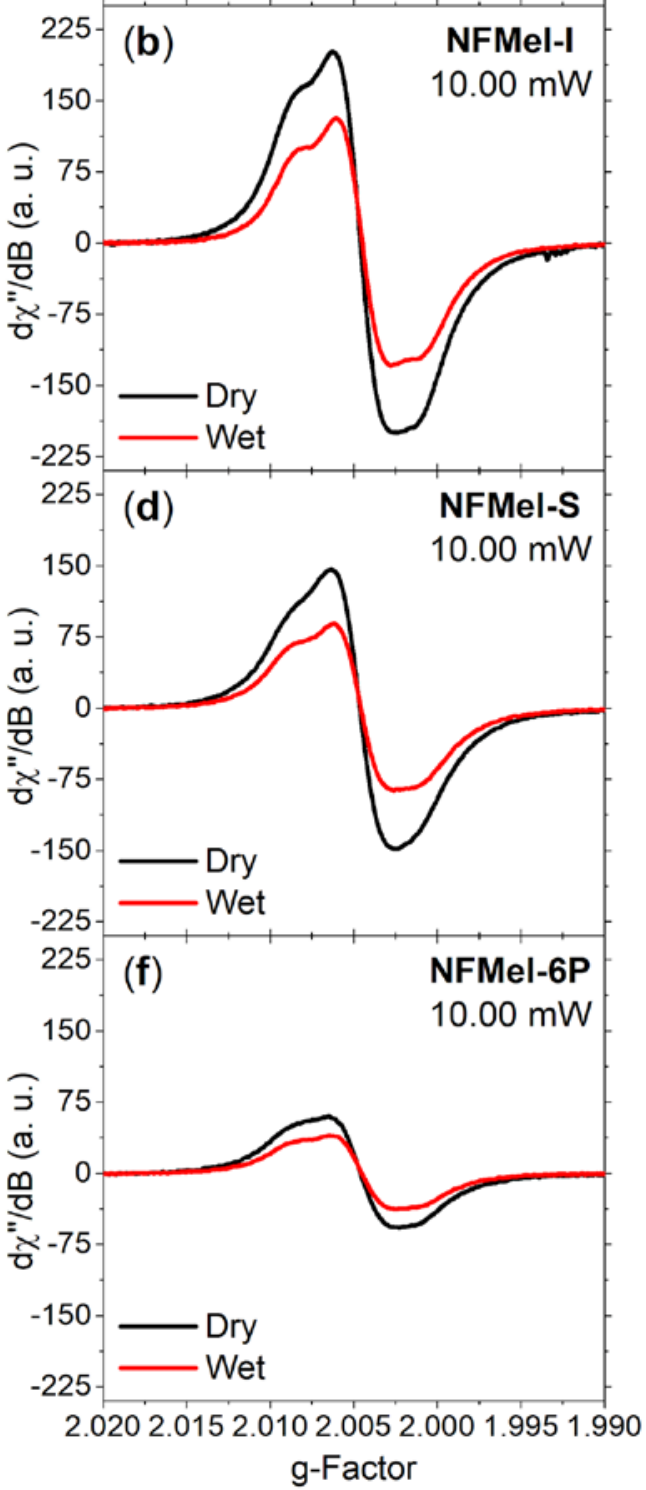

Figure S10. CW X-band EPR spectra for NF-melanin under dry and wet hydration level at low (left column) and high (right column) microwave power. 


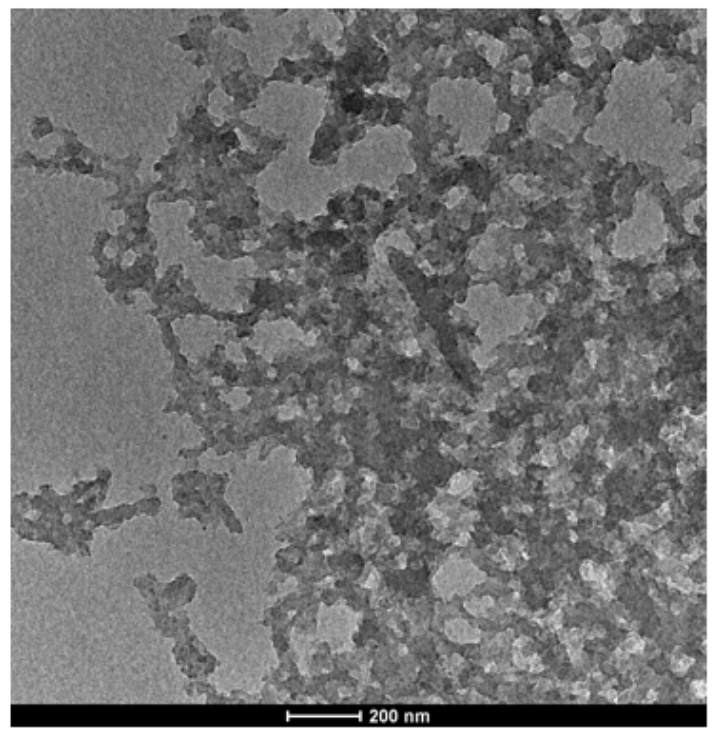

SMel

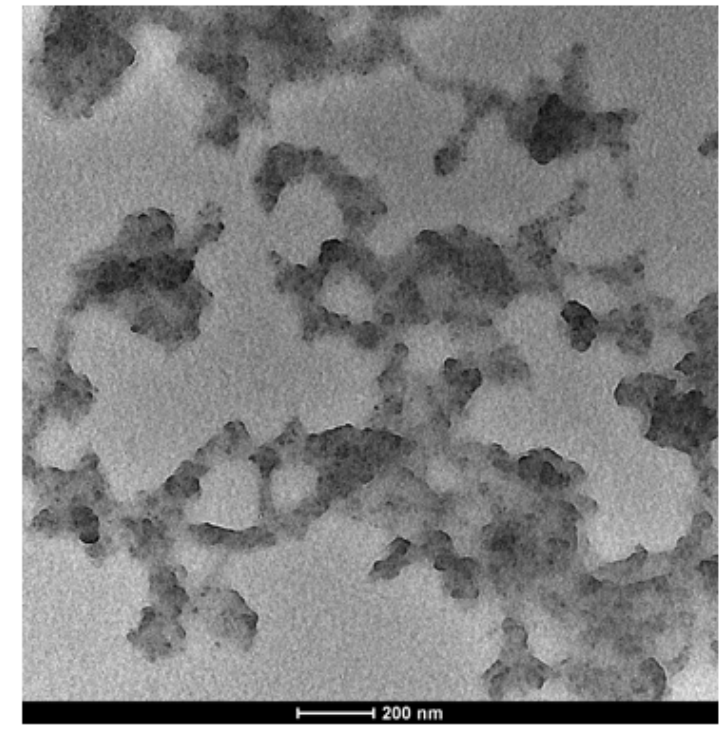

SMel-T

Figure S11. TEM images of SMel and SMel-T. The images were obtained with an FEI Tecnai G2 F20 X-Twin 200 kV microscope (LIEC, Federal University of São Carlos, Brazil).

\section{Spin density from DFT calculations}

Figure S12-S15 presents the net spin populations over the structure of S-units computed via Mulliken population analysis in a DFT/B3LYP/6-31G(d,p) approach (Orca computational software). Red and blue colors represent positive and negative relative spin densities values, respectively (a red-white-blue scale is followed). 

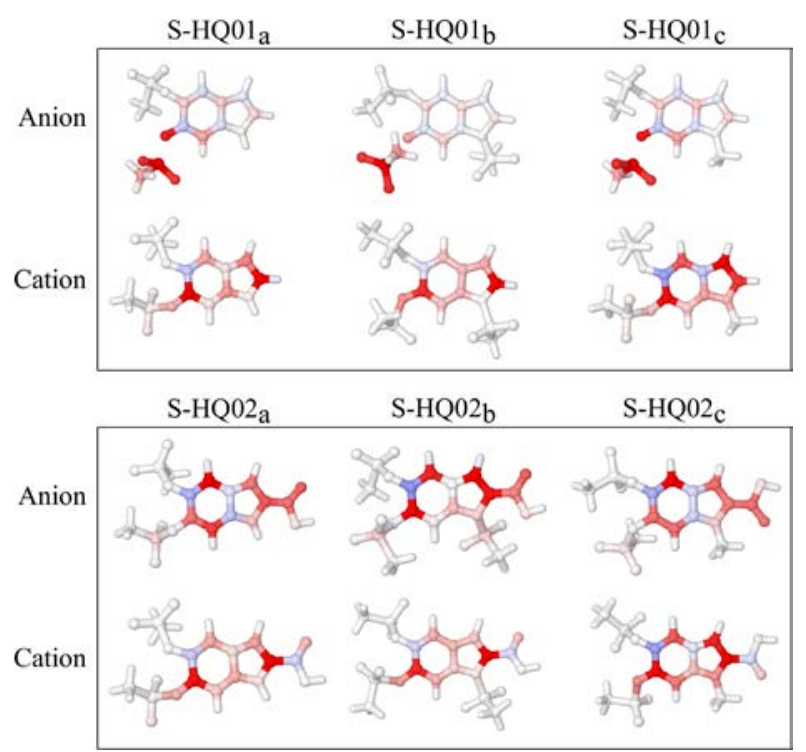

Figure S12. Representation of the spin population over the structure of S-HQ units.

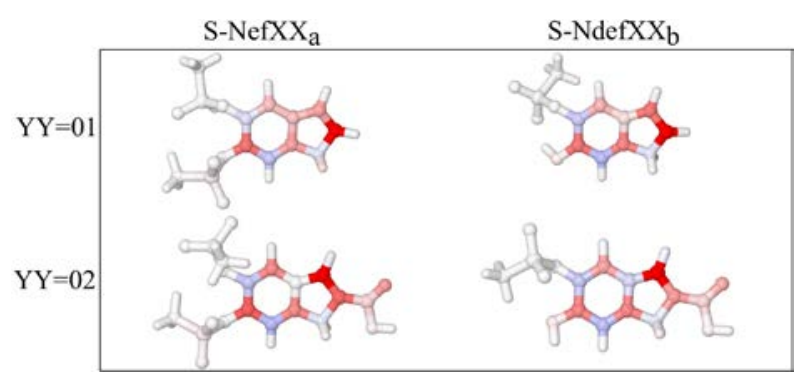

Figure S13. Representation of the spin population over the structure of S-Ndef units.

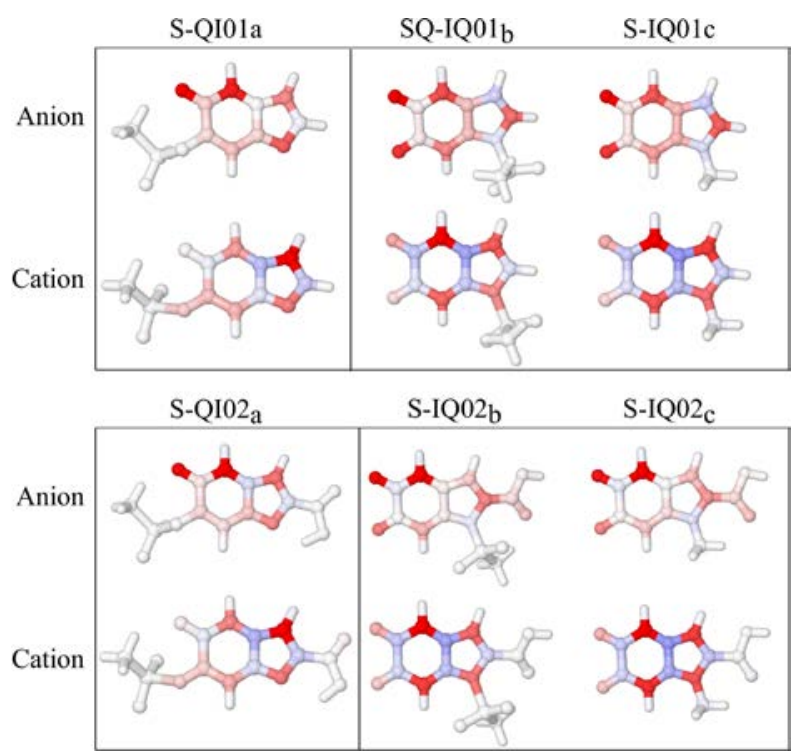

Figure S14. Representation of the spin population over the structure of S-QI and S-IQ units. 

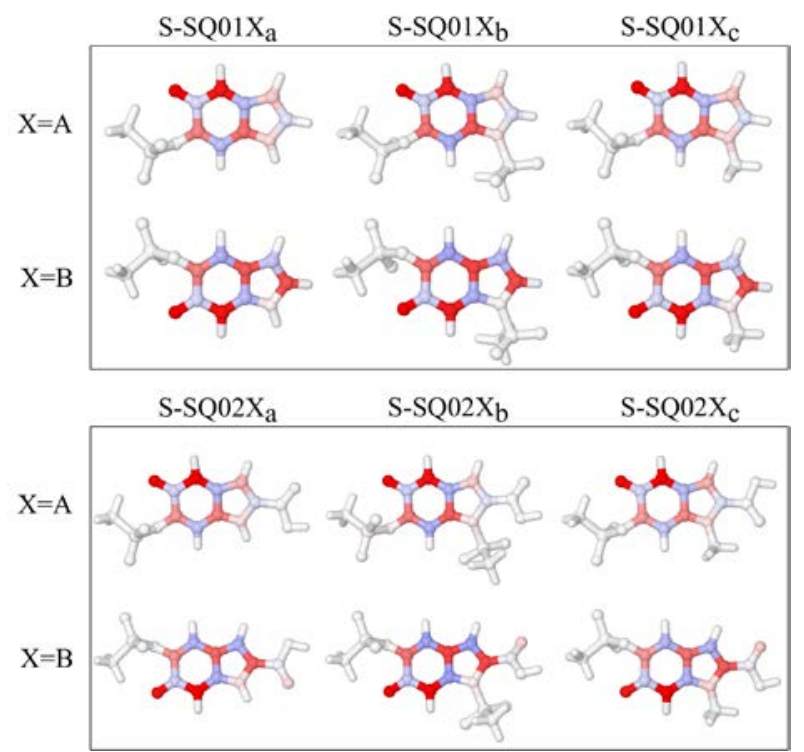

Figure S15. Representation of the spin population over the structure of S-SQ units.

\section{References:}

(R1) Felix, C. C.; Hyde, J. S.; Sarna, T.; Sealy, R. C. Interactions of Melanin with Metal Ions. Electron Spin Resonance Evidence for Chelate Complexes of Metal Ions with Free Radicals. J. Am. Chem. Soc. 1978, 100, 3922-3926.

(R2) Bronze-Uhle, E. S.; Paulin, J. V.; Piacenti-Silva, M.; Battocchio, C.; Rocco, M. L. M.; Graeff, C. F. de O. Melanin Synthesis under Oxygen Pressure. Polym. Int. 2016, 65, 1339-1346. 